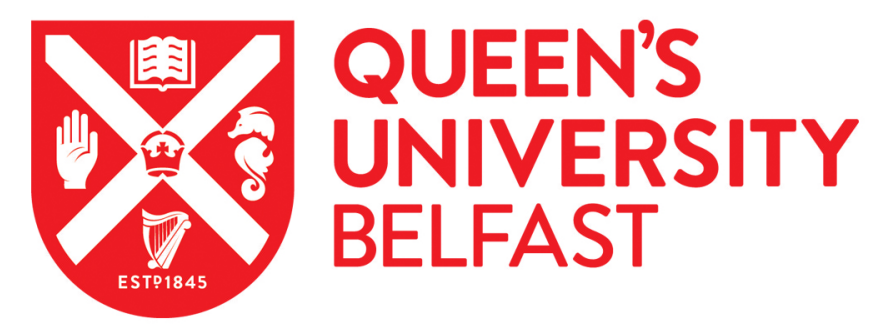

\title{
Joint Design of Reconfigurable Intelligent Surfaces and Transmit Beamforming under Proper and Improper Gaussian Signaling
}

Yu, H., Tuan, H. D., Nasir, A. A., Duong, T. Q., \& Poor, H. V. (2020). Joint Design of Reconfigurable Intelligent Surfaces and Transmit Beamforming under Proper and Improper Gaussian Signaling. IEEE Journal on Selected Areas in Communications. https://doi.org/10.1109/JSAC.2020.3007059

Published in:

IEEE Journal on Selected Areas in Communications

Document Version:

Peer reviewed version

Queen's University Belfast - Research Portal:

Link to publication record in Queen's University Belfast Research Portal

Publisher rights

Copyright 2020 IEEE. This work is made available online in accordance with the publisher's policies. Please refer to any applicable terms of use of the publisher.

\section{General rights}

Copyright for the publications made accessible via the Queen's University Belfast Research Portal is retained by the author(s) and / or other copyright owners and it is a condition of accessing these publications that users recognise and abide by the legal requirements associated with these rights.

Take down policy

The Research Portal is Queen's institutional repository that provides access to Queen's research output. Every effort has been made to ensure that content in the Research Portal does not infringe any person's rights, or applicable UK laws. If you discover content in the Research Portal that you believe breaches copyright or violates any law, please contact openaccess@qub.ac.uk. 


\title{
Joint Design of Reconfigurable Intelligent Surfaces and Transmit Beamforming under Proper and Improper Gaussian Signaling
}

\author{
H. Yu, H. D. Tuan, A. A. Nasir, T. Q. Duong, and H. V. Poor
}

\begin{abstract}
This paper considers a network of a multiple antenna array access points serving multiple single antenna downlink users with the assistance of a reconfigurable intelligent surface (RIS). The reflecting coefficients of the RIS can be programmed to ensure that the signals reflected from the RIS elements add coherently at the users. The joint design of these programmable reflecting coefficients and transmit beamforming to maximize the users' worst rate is addressed. Under either proper Gaussian signaling (PGS) or improper Gaussian signaling (IGS), the design poses a very computationally challenging nonconvex problem. Based on their exactly penalized optimization reformulation, which incorporates the computationally intractable unit-modulus constraints on the reflecting coefficients into the optimization objectives, new iterative algorithms of low computational complexity, which converge at least to a locally optimal solution, are developed. The provided simulations show not only the benefit of using RIS, but also the advantage of IGS over PGS in delivering higher rates to users.
\end{abstract}

Index Terms-Reconfigurable intelligent surface, proper and improper Gaussian signaling, transmit beamforming, phase optimization, nonconvex optimization algorithms

\section{INTRODUCTION}

The next-generation networks aim to increase 1000-fold in the average data rate, $100 \times$ improvement in the edge rate (worst data rate that a user can reasonably expect), and at least $100 \times$ decrease in energy consumption and cost compared to that offered by presently commercialized ones [1]. Though recently proposed technologies, e.g., massive multiple-input multiple-output (MIMO) and millimeter wave (mmWave) communication systems, have the potential to meet data rate requirements [2], they fail to address the target of

This work was supported in part by the National Natural Science Foundation of China (NSFC) under Grant 61901254, in part by the Institute for Computational Science and Technology, Hochiminh City, Vietnam, in part by the Australian Research Councils Discovery Projects under Grant DP190102501, in part by the U.K. Royal Academy of Engineering Research Fellowship under Grant RF1415 $\backslash 14 \backslash 22$, and in part by the U.S. National Science Foundation under Grant CCF-0939370 and Grant CCF-1908308.

Hongwen $\mathrm{Yu}$ is with the School of Electrical and Data Engineering, University of Technology Sydney, Broadway, NSW 2007, Australia (email: hongwen.yu@student.uts.edu.au), and also with the School of Communication and Information Engineering, Shanghai University, Shanghai, China (email: hongwenyu@shu.edu.cn).

Hoang D. Tuan is with the School of Electrical and Data Engineering, University of Technology Sydney, Broadway, NSW 2007, Australia (email: Tuan.Hoang@uts.edu.au).

Ali A. Nasir is with the Department of Electrical Engineering, King Fahd University of Petroleum and Minerals (KFUPM), Dhahran, Saudi Arabia (email: anasir@kfupm.edu.sa).

Trung Q. Duong is with the School of Electronics, Electrical Engineering and Computer Science, Queen's University Belfast, Belfast BT7 1NN, UK (email: trung.q.duong@qub.ac.uk).

H. Vincent Poor is with the Department of Electrical Engineering, Princeton University, Princeton, NJ 08544, USA (email: poor@ princeton.edu). low energy consumption and hardware cost [3]. Particularly, efficient communication by these technologies require large number of costly and power-hungry radio frequency (RF) chains (depending on the number of antennas), where each comprises several active components. Therefore, researchers are still hunting for an energy efficient as well as spectral efficient solution to assist the realization of futuristic networks [4], [5].

Recently, the use of reconfigurable intelligent surface (RIS) has been identified as a low-energy consumption and spectral efficient solution [6]-[8]. RIS is a planar array of a large number of low-cost and nearly-passive reflecting elements with reconfigurable parameters. Each reflecting element on RIS can introduce an independent phase shift on the incident electromagnetic wave (from the access points (AP) or transmitter) [9]. The phase shifts induced by the passive elements can be programmed to ensure that reflected signals from the RIS elements coherently add, together or also with other directpath signals, if available, at the user end [7], [10], [11]. More importantly, RIS can be installed in such places such as buildings which block the direct transmission from the AP to its users [12]. RIS technology is quite different with several distinct positives when compared with the other existing technologies such as backscatter communication [13], [14], amplify-and forward (AF) relaying, and intelligent surface based massive MIMO [15]. A detailed comparison among these technologies is provided in [8], [16]. The work [17] shows that a particular RIS-aided MIMO system can achieve the same rate performance as that achieved by massive MIMO system without using RIS, but the former option is energy-and cost-efficient with significantly reduced active antennas/RF chains.

Naturally, RIS-aided systems need to be optimized in terms of transmit beamformers and RIS reflecting coefficients for delivering high rates. Optimization of RIS-aided systems looks computationally intractable because of two reasons: $(i)$ both rate and transmit power become very complex functions in the beamformers and RIS reflecting coefficients; (ii) The RIS reflecting coefficients are constrained by the nonconvex unitmodulus constraint (UMC). Alternating optimization between the beamformers and the RIS reflecting coefficients is often applied. Each round of alternating optimization consists of optimization in the beamformers with the reflecting coefficients held fixed and optimization in the reflecting coefficients with the beamformers held fixed. These optimization problems are still nonconvex and thus still computationally challenging. The 
authors in [6] and [18] use general-purpose gradient/projected gradient algorithms for their computation, which do not necessarily converge. The authors in [17] reformulate alternating optimization in the reflecting coefficients as a matrix rankone constrained optimization problem. The matrix rank-one constraint is then dropped for convex relaxation. The reader is also referred to [19] for computational efficiency of this convex relaxation. At each round of alternating optimization, the objective function is replaced by a surrogate function in [20]-[22], and then the nonconvex unit-modulus constraint on the reflecting coefficients is relaxed to the convex boundedby-unit-modulus constraint for alternating optimization in the reflecting coefficients, while the minimum-mean-square-error (MMSE) algorithm is used for alternating optimization in the beamformers. Alternating optimization does not seem to be computationally efficient if each round still invokes two nonconvex problems, which are still computationally challenging. Theoretically, its found solution is not even locally optimal as it is only optimal in one set of variables with other set of variables held fixed.

It should be emphasized that all the aforementioned works are based on the conventional proper Gaussian signaling (PGS), which is induced by linearly beamforming proper Gaussian source. Recently, it has been shown e.g. in [23][30] that improper Gaussian signaling (IGS), which is induced by widely linearly beamforming proper Gaussian source [31], outperforms PGS clearly in interference-limited networks. Under PGS, the transmit signal is still proper Gaussian and completely characterized by its covariance. In contrast, the transmit signal under IGS is improper Gaussian and is characterized by the so-called augmented covariance of double size with a special structure, which involves not only its covariance but also its pseudo-covariance [31]. As such, in contrast to PGS, which is induced by single beamformers, IGS is induced by pairs of correlated beamformers. The design of beamforming vectors for IGS is more complex than for PGS because it involves twice the number of decision variables, and more importantly, the rate functions are log-determinant $\log \operatorname{det}($.$) even for multi-input single output (MISO) networks.$ Their optimization is much more computationally challenging than that for PGS, which involves logarithmic functions only.

Against the above background, this paper investigates the joint design of transmit beamformers and RIS reflecting coefficient in networks of a multiple antenna array AP serving multiple single-antenna users with the aid of an RIS, under both PGS and IGS. The contributions of the paper are following:

- Under PGS, based on the exactly penalized optimization reformulation, which incorporates the computationally intractable unit-modulus constraint on the reflecting coefficients into the optimization objective, we develop an algorithm of low computational complexity, each iteration of which invokes up to two convex problems. Moreover, it rapidly converges at least to a locally optimal solution.

- This is the first work to use IGS for RIS-aided communication networks. Again, based on the exactly penalized optimization reformulation, we develop another algorithm of low computational complexity, which rapidly con- verges at least to a locally optimal solution.

- IGS is shown to outperform PGS clearly in severely interference-limited scenarios when the number of transmit antennas is less than the number of served users.

The paper is organized as follows. The joint design of beamformers and RIS reflecting coefficients to maximize the worst users' rate under PGS and RIS are addressed in Sections II and Section III, respectively. The simulations to demonstrate the advantage of RIS over PGS are provided in Section IV, which is followed by Section $\mathrm{V}$ for concluding the paper. The Appendix provides fundamental matrix inequalities, which were used for developing the algorithms in Sections II and III.

Notation. Only the design vector/matrix variable are printed in boldface. $[X]^{2}$ is $X X^{H}$, and $\langle X, Y\rangle=\operatorname{trace}\left(X^{H} Y\right)$ for the matrices $X$ and $Y$. Accordingly, the Frobenius norm of $X$ is defined by $\|X\|=\sqrt{\operatorname{trace}\left(X^{H} X\right)}$. We also write $\langle X\rangle=\operatorname{trace}(X)$ for notational simplicity. The notation $X \succeq 0$ ( $X \succ 0$, resp.) used for the Hermitian symmetric matrix $X$ indicates that it is positive definite (positive semidefinite, resp.), and accordingly, $X \succeq 0$ is called a semidefinite (convex) constraint. $I_{N}$ is the identity matrix of size $N \times N$, while $O_{M \times N}$ is zero matrix of size $M \times N$. In symmetric block matrices or long matrix expressions, we use $*$ as an ellipsis for terms that are induced by symmetry, e.g. $\left(X+(*)^{H}\right) \equiv\left(X+X^{H}\right)$, and

$$
\left[\begin{array}{cc}
\left(X+(*)^{H}\right)+B & * \\
A & Y+(*)^{H}
\end{array}\right] \equiv\left[\begin{array}{cc}
\left(X+X^{H}\right)+B & A^{H} \\
A & Y+Y^{H}
\end{array}\right] .
$$

Lastly, denote by $\mathcal{C}(0, a)$ the set of circular Gaussian random variable with the zero means and variance $a$.

\section{Proper GAUSSiAn Signaling}

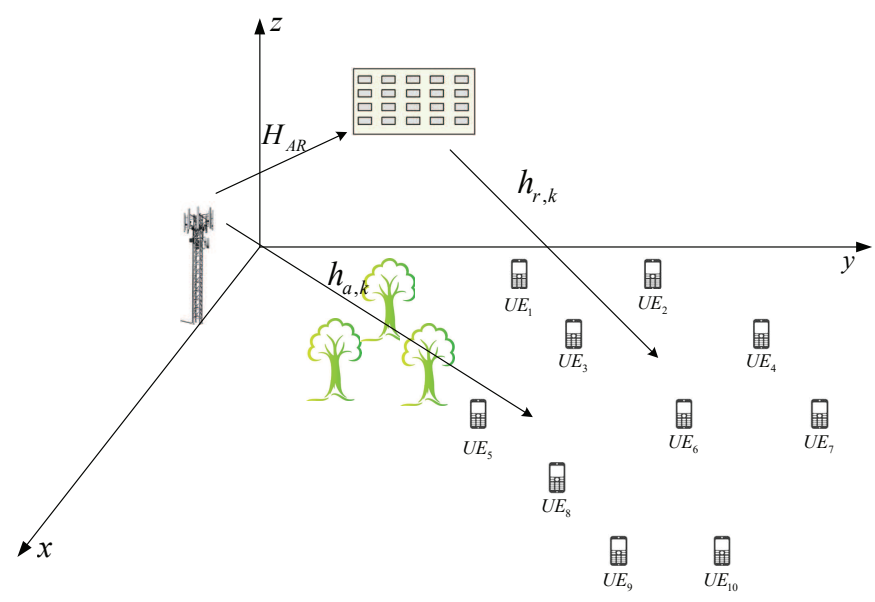

Fig. 1: System model

Consider a RIS-aided network as illustrated by Fig. 1, where a RIS of $N$ reflecting units assists the downlink from an $M$ antenna array AP to $K$ single-antenna users (UEs). Let $x$ be the transmit signal from the AP. The received signal at UE $k$ can be expressed as

$y_{k}=\left(\sqrt{\beta_{\mathrm{AP}-\mathrm{RIS}}} \sqrt{\beta_{\mathrm{RIS}-\mathrm{k}}} h_{r, k} \mathbf{R}_{\mathrm{RIS}-\mathrm{k}}^{1 / 2} \boldsymbol{\Theta} H_{A R}+\sqrt{\beta_{\mathrm{AP}-\mathrm{k}}} h_{a, k}\right) x+n_{k}$, 
where $\sqrt{\beta_{\text {AP-RIS }}}$ and $\sqrt{\beta_{\text {RIS-k }}}$ model the path-loss and largescale fading of the AP-to-RIS link and from the RIS-to-UE $k$ link, respectively [18], [32], $\sqrt{\beta_{\mathrm{AP}-\mathrm{k}}}$ models the path-loss and large-scale fading of the direct path between the AP and the UE $k, H_{A R} \in \mathbb{C}^{N \times M}$ is the line-of-sight (LoS) channel matrix between the AP and RIS, $h_{r, k} \in \mathbb{C}^{1 \times N}$ and $h_{a, k} \in \mathbb{C}^{1 \times M},{ }^{1}$ respectively, denote the small-scale fading channels from the RIS and the AP to UE $k, \mathbf{R}_{\mathrm{RIS}-\mathrm{k}} \in \mathbb{C}^{N \times N}$ represents the spatial correlation matrix for the RIS elements with respect to the user $k$ [18], [33], $n_{k} \in \mathcal{C}(0, \sigma)$ is the background noise at UE $k$, and for $\boldsymbol{\theta} \triangleq\left(\theta_{1}, \ldots, \theta_{N}\right) \in \mathbb{C}^{N}$ with

$$
\left|\theta_{n}\right|=1, n=1, \ldots, N
$$

which denotes the vector of the RIS's reflecting-coefficients, the matrix of reflection-coefficients of the RIS is

$$
\boldsymbol{\Theta}=\left[\begin{array}{cccc}
\theta_{1} & 0 & \cdots & 0 \\
0 & \theta_{2} & \cdots & 0 \\
0 & 0 & \cdots & \theta_{N}
\end{array}\right] \in \mathbb{C}^{N \times N}
$$

Since the RIS is usually deployed on the facade of high-rise building [12] and the AP is usually deployed at a certain height it is justified to assume LoS communication between the AP and RIS [18]. The communication channel between the AP and UEs $h_{a, k}$ is non-LOS (NLoS) and thus modeled by Rayleigh fading, while the presence of LoS link is assumed between the RIS and UEs and thus the corresponding channel $h_{r, k}$ is modeled by Rician fading [34]. The NLoS communication between the AP and UEs motivates the use of an RIS to support the transmission. To focus on the design of beamforming vectors and reflection-coefficients of the RIS, the paper assumes that the channel state information is perfectly available at the AP, which is responsible for calculating the reflection-coefficients of the RIS and feeding them back to the RIS controller through dedicated control channels. This assumption is in line with the existing relevant research in the literature [20]-[22]. Under this assumption, the results of the paper will represent an upper bound on the practical achievable performance.

Let $s_{k} \in \mathcal{C}(0,1)$ be the information intended for UE $k$. Under PGS, the proper Gaussian source $s_{k}$ is linearly beamformed by the beamformer $\mathbf{w}_{k} \in \mathbb{C}^{M}$. Therefore, the transmit signal $x$, which is given by

$$
x=\sum_{k=1}^{K} \mathbf{w}_{k} s_{k}
$$

is also proper Gaussian. Using (3), the equation (1) is written by

$$
y_{k}=\mathcal{H}_{k}(\boldsymbol{\theta}) \sum_{k=1}^{K} \mathbf{w}_{k} s_{k}+n_{k}
$$

for

$$
\begin{aligned}
\mathcal{H}_{k}(\boldsymbol{\theta}) \triangleq \sqrt{\beta_{\mathrm{AP}-\mathrm{RIS}}} & \sqrt{\beta_{\mathrm{RIS}-\mathrm{k}}} h_{r, k} \mathbf{R}_{\mathrm{RIS}-\mathrm{k}}^{1 / 2} \boldsymbol{\Theta} H_{A R} \\
& +\sqrt{\beta_{\mathrm{AP}-\mathrm{k}}} h_{a, k} \in \mathbb{C}^{1 \times M} .
\end{aligned}
$$

\footnotetext{
${ }^{1}$ In Section IV, we also consider particular cases of no direct path between the AP and UEs, i.e. $h_{a, k} \equiv 0$
}

Let $\mathbf{w} \triangleq\left\{\mathbf{w}_{k}, k \in \mathcal{K}\right\}$. Based on the signal-to-interferenceplus-noise (SINR) defined by

$$
\rho_{k}(\boldsymbol{\theta}, \mathbf{w}) \triangleq \frac{\left|\mathcal{H}_{k}(\boldsymbol{\theta}) \mathbf{w}_{k}\right|^{2}}{\sum_{j \in \mathcal{K} \backslash\{k\}}\left|\mathcal{H}_{k}(\boldsymbol{\theta}) \mathbf{w}_{j}\right|^{2}+\sigma},
$$

the rate in nats at $\mathrm{UE} k$ is calculated by

$$
r_{k}(\boldsymbol{\theta}, \mathbf{w})=\ln \left(1+\rho_{k}(\boldsymbol{\theta}, \mathbf{w})\right) .
$$

Given a power budget $P$, the max-min rate optimization is then formulated as

$$
\begin{array}{r}
\max _{\boldsymbol{\theta}, \mathbf{w}} \min _{k=1, \ldots, K} r_{k}(\boldsymbol{\theta}, \mathbf{w}) \quad \text { s.t. } \quad(2), \\
\sum_{k=1}^{K}\left\|\mathbf{w}_{k}\right\|^{2} \leq P,
\end{array}
$$

which is equivalent to the following problem of max-min SINR optimization:

$$
\max _{\boldsymbol{\theta}, \mathbf{w}} f(\boldsymbol{\theta}, \mathbf{w}) \triangleq \min _{k=1, \ldots, K} \rho_{k}(\boldsymbol{\theta}, \mathbf{w}) \quad \text { s.t. } \quad(2),(8 b) .
$$

This optimization problem is nonconvex because its objective function is nonconcave and the unit-modulus constraint (UMC) (2) is obviously nonconvex. To the authors' best knowledge, there is no efficient method to handle the UMC (2), which is often relaxed to the convex bounded-by-unit-modulus constraint

$$
\left|\theta_{n}\right|^{2} \leq 1, n=1, \ldots, N .
$$

The existing works use alternating optimization to address (8). Let $\left(\theta^{(\kappa)}, w^{(\kappa)}\right)$ be a feasible point for (8) that is found from the $(k-1)$-th round. The $n$-th round aims to solve the following alternating optimization problem in $\mathbf{w}$ to generate the next iterative point $w^{(\kappa+1)}$ :

$$
\max _{\mathbf{w}} f\left(\theta^{(\kappa)}, \mathbf{w}\right) \quad \text { s.t. } \quad(8 b) .
$$

and then aims to solve the following alternating optimization problem in $\boldsymbol{\theta}$ to generate the next iterative point $\theta^{(\kappa+1)}$ :

$$
\max _{\boldsymbol{\theta}} f\left(\boldsymbol{\theta}, w^{(\kappa+1)}\right) \quad \text { s.t. } \quad(2),
$$

It should be noted that the SINR $\rho_{k}$ defined by (6) is a quotient of two functions, which are separately convex quadratic in $\boldsymbol{\theta}$ and $\mathbf{w}$, so both (11) and the unit-modulusrelaxed problem

$$
\max _{\boldsymbol{\theta}} f\left(\boldsymbol{\theta}, w^{(\kappa)}\right) \quad \text { s.t. } \quad(10)
$$

can be efficiently computed by the algorithms of [35], [36].

In [20], the objective is replaced by a surrogate function at each round so the alternating optimization in $\mathbf{w}$ is a convex problem, and by relaxing the UMC (2) by (10), the alternating optimization in $\boldsymbol{\theta}$ is also a convex problem.

The authors in [17] consider the following problem of power minimization subject to the SINR constraints

$$
\min _{\boldsymbol{\theta}, \mathbf{w}} \sum_{k=1}^{K}\left\|\mathbf{w}_{k}\right\|^{2} \quad \text { s.t. } \quad(2), \rho_{k}(\boldsymbol{\theta}, \mathbf{w}) \geq \gamma, k \in \mathcal{K},
$$

for a given $\gamma>0$. The alternating optimization in $\mathbf{w}$ to generate $w^{(\kappa+1)}$ is equivalent to a second-order cone problem 
of convex programming [37]. The alternating optimization in $\boldsymbol{\theta}$ to generate $\theta^{(\kappa+1)}$ is the feasibility problem

$$
(2), \rho_{k}\left(\boldsymbol{\theta}, w^{(\kappa+1)}\right) \geq \gamma, k \in \mathcal{K} .
$$

The authors use the auxiliary matrix variable $\widetilde{\Theta} \triangleq\left[\begin{array}{cc}\Theta & \theta \\ \boldsymbol{\theta}^{H} & 1\end{array}\right] \in$ $\mathbb{C}^{(N+1) \times(N+1)}, \Theta \in \mathbb{C}^{N \times N}$, which must satisfy the semidefinite constraint $\widetilde{\Theta} \succeq 0$ and linear constraints $\widetilde{\Theta}(n, n)=1$, $n \in \mathcal{N}$ and the matrix rank-one constraint

$$
\operatorname{rank}(\widetilde{\Theta})=1 .
$$

This matrix rank-one constraint is then dropped to have a convex relaxation for the feasibility problem (15). Obviously, $\theta^{(\kappa)}$ is already feasible for (15), so it is not clear for what one needs to consider (15) and how to judge which of feasible points for (15) is preferred. The number of decision variables in the convex relaxed problem is $N(2 N+3) / 2$, which is quickly grown in $N$. For instance, it is already 2575 for $N=50$, hiking the computational complexity $\mathcal{O}\left((N(2 N+2) / 2)^{3}\right)$ of convex solvers. The reader is also referred to [19] for capacity of convex relaxation-based approaches in locating the needed matrix-rank one solution. After all, like [20], the convergence of the alternating procedure in [17] is not guaranteed.

We now propose a quite different approach for addressing the max-min SINR optimization problem (9). Note that the UMC (2) is equivalent to the convex constraint (10) plus the constraint

$$
N \leq \sum_{n=1}^{N}\left|\theta_{n}\right|^{2},
$$

which is reverse convex [38]. Indeed, (10) implies $\sum_{n=1}^{N}\left|\theta_{n}\right|^{2} \leq N$, which together with (17) yield $\sum_{n=1}^{N}\left|\theta_{n}\right|^{2}=N$ that is possible if and only if (2) is fulfilled. It is obvious that (17) is the same as

$$
\frac{1}{N}-\frac{1}{\sum_{n=1}^{N}\left|\theta_{n}\right|^{2}} \geq 0
$$

and the equality sign in (18) forces the UMC (2). This means

$$
\frac{1}{N}-\frac{1}{\sum_{n=1}^{N}\left|\theta_{n}\right|^{2}}
$$

can be used a measure for satisfaction of the UCM (2). Like [39]-[41], instead of handling the nonconvex constraint (18) we minimize the measure (19) for its satisfaction by incorporating it in the optimization objective, leading to the following exactly penalized optimization problem

$$
\max _{\boldsymbol{\theta}, \mathbf{w}} g(\boldsymbol{\theta}, \mathbf{w}) \triangleq\left[f(\boldsymbol{\theta}, \mathbf{w})+\mu\left(\frac{1}{N}-\frac{1}{\sum_{n=1}^{N}\left|\theta_{n}\right|^{2}}\right)\right]
$$

where $\mu>0$ is the penalty parameter. ${ }^{2}$ For $\mu$ sufficiently large, (9) and (20) have the same optimal solution [42]. Later, we will show how $\mu$ is chosen before hand.

Although all constraints in (20) are convex, (20) is still a difficult nonconvex problem as its objective remains to

\footnotetext{
${ }^{2}$ Since the constraints ( $\left.8 b\right)$ and $(8 b)$ are already convex, there is no need to incorporate them in the optimization objective.
}

be nonconcave. We now develop iterative processes for its computation.

Let $\left(w^{(\kappa)}, \theta^{(\kappa)}\right)$ be the feasible point for (20) that is found from the $(\kappa-1)$-th round.

\section{A. Alternating descent round}

In alternating descent, we generate the next iterative point $w^{(\kappa+1)}$ with $\boldsymbol{\theta}$ held fixed at $w^{(\kappa)}$ and then the next iterative point $\theta^{(\kappa+1)}$ is generated with $\mathbf{w}$ held fixed at $w^{(\kappa+1)}$.

1) Beamforming descent iteration: To generate $w^{(\kappa+1)}$ we do not solve (11) but we seek $w^{(\kappa+1)}$ such that

$$
f\left(\theta^{(\kappa)}, w^{(\kappa+1)}\right)>f\left(\theta^{(\kappa)}, w^{(\kappa)}\right) .
$$

Using the inequality (73) in the appendix A yields

$$
\begin{aligned}
\rho_{k}\left(\theta^{(\kappa)}, \mathbf{w}\right) & \geq \rho_{k}^{(\kappa)}(\mathbf{w}) \\
& \triangleq 2 \Re\left\{b_{k}^{(\kappa)} \mathbf{w}_{k}\right\}-c_{k}^{(\kappa)} \sum_{j \in \mathcal{K} \backslash\{k\}}\left|\mathcal{H}_{k}\left(\theta^{(\kappa)}\right) \mathbf{w}_{j}\right|^{2} \\
& \\
&
\end{aligned}
$$

with

$$
\begin{gathered}
b_{k}^{(\kappa)} \triangleq \frac{\left(w_{k}^{(\kappa)}\right)^{H}\left(\mathcal{H}_{k}\left(\theta^{(\kappa)}\right)\right)^{H} \mathcal{H}_{k}\left(\theta^{(\kappa)}\right)}{y_{k}^{(\kappa)}}, \\
0<c_{k}^{(\kappa)} \triangleq \frac{\left|\mathcal{H}_{k}\left(\theta^{(\kappa)}\right) w_{k}^{(\kappa)}\right|^{2}}{\left(y_{k}^{(\kappa)}\right)^{2}}, \\
0<y_{k}^{(\kappa)} \triangleq \sum_{j \in \mathcal{K} \backslash\{k\}}\left|\mathcal{H}_{k}\left(\theta^{(\kappa)}\right) w_{j}^{(\kappa)}\right|^{2}+\sigma .
\end{gathered}
$$

The function $\rho_{k}^{(\kappa)}(\mathbf{w})$ is seen quadratic concave, which matches with $\rho_{k}\left(\theta^{(\kappa)}, \mathbf{w}\right)$ at $w^{(\kappa)}$. We solve the following convex problem of computational complexity $\mathcal{O}\left((M K)^{3}\right)$ $\left[43\right.$, p. 4] at the $\kappa$-th iteration to generate $w^{(\kappa+1)}$ :

$$
\max _{\mathbf{w}} f^{(\kappa)}(\mathbf{w}) \triangleq \min _{k=1, \ldots, K} \rho_{k}^{(\kappa)}(\mathbf{w}) \quad \text { s.t. } \quad(8 b),
$$

where $f^{(\kappa)}$ is seen concave as a minimum of concave functions [38].

Note that

$$
f^{(\kappa)}\left(w^{(\kappa+1)}\right)>f^{(\kappa)}\left(w^{(\kappa)}\right)
$$

as far as $w^{(\kappa+1)} \neq w^{(\kappa)}$ because $w^{(\kappa+1)}$ is the optimal solution of (23) while $w^{(\kappa)}$ is only a feasible point. Therefore,

$$
\begin{aligned}
f\left(\theta^{(\kappa)}, w^{(\kappa+1)}\right) & \geq f^{(\kappa)}\left(w^{(\kappa+1)}\right) \\
& >f^{(\kappa)}\left(w^{(\kappa)}\right) \\
& =f\left(\theta^{(\kappa)}, w^{(\kappa)}\right),
\end{aligned}
$$

showing (21).

2) Phase descent iteration: We seek the next iterative point $\theta^{(\kappa+1)}$ such that

$$
g\left(\theta^{(\kappa+1)}, w^{(\kappa+1)}\right)>g\left(\theta^{(\kappa)}, w^{(\kappa+1)}\right) .
$$

Using the inequality (73) in the appendix A yields

$$
\begin{aligned}
\rho_{k}\left(\boldsymbol{\theta}, w^{(\kappa+1)}\right) & \geq \tilde{\rho}_{k}^{(\kappa)}(\boldsymbol{\theta}) \\
& \triangleq 2 \Re\left\{\tilde{b}_{k}^{(\kappa)} \mathcal{H}_{k}(\boldsymbol{\theta}) w_{k}^{(\kappa+1)}\right\}
\end{aligned}
$$




$$
\begin{aligned}
\left\langle\boldsymbol{X}_{k}, \sum_{j \in \mathcal{K} \backslash\{k\}} \mathbf{Y}_{j}\right\rangle= & \left\langle\boldsymbol{X}_{k}+\epsilon I_{M}, \sum_{j \in \mathcal{K} \backslash\{k\}} \mathbf{Y}_{j}+\epsilon I_{M}\right\rangle-\epsilon\left\langle\boldsymbol{X}_{k}+\sum_{j \in \mathcal{K} \backslash\{k\}} \mathbf{Y}_{j}\right\rangle-\epsilon^{2} M \\
\leq & \frac{1}{2}\left[\left\|\left(X_{k}^{(\kappa)}(\epsilon)\right)^{-1 / 2}\left(\boldsymbol{X}_{k}+\epsilon I_{M}\right)\left(Y_{\backslash k}^{(\kappa)}(\epsilon)\right)^{1 / 2}\right\|^{2}\right. \\
& \left.+\left\|\left(X_{k}^{(\kappa)}(\epsilon)\right)^{1 / 2}\left(\sum_{j \in \mathcal{K} \backslash\{k\}} \mathbf{Y}_{j}+\epsilon I_{M}\right)\left(Y_{\backslash k}^{(\kappa)}(\epsilon)\right)^{-1 / 2}\right\|^{2}\right] \\
& -\epsilon\left\langle\boldsymbol{X}_{k}+\sum_{j \in \mathcal{K} \backslash\{k\}} \mathbf{Y}_{j}\right\rangle-\epsilon^{2} M \\
\triangleq & g_{k}^{(\kappa)}\left(\mathbf{w}, \boldsymbol{X}_{k}, \mathbf{Y}\right),
\end{aligned}
$$

$$
\left[\begin{array}{cc}
\left(\mathcal{H}_{k}\left(\theta^{(\kappa)}\right) Y_{k}^{(\kappa)}(\eta) \mathcal{H}_{k}^{H}(\boldsymbol{\theta})+(*)^{H}\right)-\mathbf{z}_{k}-\eta\left\langle\boldsymbol{X}_{k}\right\rangle & * \\
Y_{k}^{(\kappa)}(\eta) \mathcal{H}_{k}^{H}\left(\theta^{(\kappa)}\right) & {\left[w_{k}^{(\kappa)}\left(\mathbf{w}_{k}\right)^{H}+(*)^{H}\right]-\left[w_{k}^{(\kappa)}\right]^{2}+\eta I_{M}}
\end{array}\right] \succeq 0
$$

$$
-\tilde{c}_{k}^{(\kappa)} \sum_{j \in \mathcal{K} \backslash\{k\}}\left|\mathcal{H}_{k}(\boldsymbol{\theta}) w_{j}^{(\kappa+1)}\right|^{2}-\sigma \tilde{c}_{k}^{(\kappa)}(25)
$$

with

$$
\begin{gathered}
\tilde{b}_{k}^{(\kappa)} \triangleq \frac{\left(w_{k}^{(\kappa+1)}\right)^{H}\left(\mathcal{H}_{k}\left(\theta^{(\kappa)}\right)\right)^{H}}{y_{k}^{(\kappa+1)}}, \\
0<\tilde{c}_{k}^{(\kappa)} \triangleq \frac{\left|\mathcal{H}_{k}\left(\theta^{(\kappa)}\right) w_{k}^{(\kappa+1)}\right|^{2}}{\left(y_{k}^{(\kappa+1)}\right)^{2}}, \\
0<y_{k}^{(\kappa+1)} \triangleq \sum_{j \in \mathcal{K} \backslash\{k\}}\left|\mathcal{H}_{k}\left(\theta^{(\kappa)}\right) w_{j}^{(\kappa+1)}\right|^{2}+\sigma,
\end{gathered}
$$

and

$$
\begin{aligned}
\frac{1}{\sum_{n=1}^{N}\left|\theta_{n}\right|^{2}} & \leq \iota^{(\kappa)}(\boldsymbol{\theta}) \\
& \triangleq \frac{1}{\sum_{n=1}^{N}\left(2 \Re\left\{\left(\theta_{n}^{(\kappa)}\right) * \theta_{n}\right\}-\left|\theta_{n}^{(\kappa)}\right|^{2}\right)}
\end{aligned}
$$

over the trust region

$$
\sum_{n=1}^{N}\left(2 \Re\left\{\left(\theta_{n}^{(\kappa)}\right)^{*} \theta_{n}\right\}-\left|\theta_{n}^{(\kappa)}\right|^{2}\right)>0
$$

We solve the following optimization problem of computational complexity $\mathcal{O}\left((N)^{3}(N+1)\right)[43$, p. 4] at the $\kappa$-th iteration to generate $\theta^{(\kappa+1)}$ :

$$
\max _{\boldsymbol{\theta}} g^{(\kappa)}(\boldsymbol{\theta}) \triangleq\left[\min _{k=1, \ldots, K} \tilde{\rho}_{k}^{(\kappa)}(\boldsymbol{\theta})+\mu\left(\frac{1}{N}-\iota^{(\kappa)}(\boldsymbol{\theta})\right)\right]
$$

which is convex because its objective function is concave. Note that $g\left(\boldsymbol{\theta}, w^{(\kappa+1)}\right) \geq g^{(\kappa)}(\boldsymbol{\theta})$, and $g\left(\theta^{(\kappa)}, w^{(\kappa+1)}\right)=$ $g^{(\kappa)}\left(\theta^{(\kappa)}\right)$, so, by using a similar argument to that for proving (54), we can show (24) as far as $\theta^{(\kappa+1)} \neq \theta^{(\kappa)}$.

3) Convergence: It follows from (21) and (24) that

$$
g\left(\theta^{(\kappa+1)}, w^{(\kappa+1)}\right)>g\left(\theta^{(\kappa)}, w^{(\kappa)}\right),
$$

and as such the sequence $\left\{\left(\theta^{(\kappa)}, w^{(\kappa)}\right)\right\}$ converges to a point $(\bar{\theta}, \bar{w})$ such that $\bar{\theta}(\bar{w}$, resp.) is a locally optimal solution of (20) with $\mathbf{w}(\boldsymbol{\theta}$, resp.) held fixed at $\bar{w}(\bar{\theta}$, resp.).

\section{B. Path-following iteration}

Using the inequality (72) in the appendix A yields

$$
\rho_{k}(\boldsymbol{\theta}, \mathbf{w}) \geq a_{k}^{(\kappa)}-\frac{b_{k}^{(\kappa)}}{\left|\mathcal{H}_{k}(\boldsymbol{\theta}) \mathbf{w}_{k}\right|^{2}}-c_{k}^{(\kappa)} \sum_{j \in \mathcal{K} \backslash\{k\}}\left|\mathcal{H}_{k}(\boldsymbol{\theta}) \mathbf{w}_{j}\right|^{2}
$$

where

$$
\begin{gathered}
a_{k}^{(\kappa)} \triangleq \frac{3\left|\mathcal{H}_{k}\left(\theta^{(\kappa)}\right) w_{k}^{(\kappa)}\right|^{2}}{y_{k}^{(\kappa)}}, \\
0<b_{k}^{(\kappa)} \triangleq \frac{\left|\mathcal{H}_{k}\left(\theta^{(\kappa)}\right) w_{k}^{(\kappa)}\right|^{4}}{y_{k}^{(\kappa)}}, 0<c_{k}^{(\kappa)} \triangleq \frac{\left|\mathcal{H}_{k}\left(\theta^{(\kappa)}\right) w_{k}^{(\kappa)}\right|^{2}}{\left(y_{k}^{(\kappa)}\right)^{2}}, \\
0<y_{k}^{(\kappa)} \triangleq \sum_{j \in \mathcal{K} \backslash\{k\}}\left|\mathcal{H}_{k}\left(\theta^{(\kappa)}\right) w_{j}^{(\kappa)}\right|^{2}+\sigma .
\end{gathered}
$$

We have

$$
\begin{aligned}
\sum_{j \in \mathcal{K} \backslash\{k\}}\left|\mathcal{H}_{k}(\boldsymbol{\theta}) \mathbf{w}_{j}\right|^{2} & =\left\langle\left[\mathcal{H}_{k}^{H}(\boldsymbol{\theta})\right]^{2}, \sum_{j \in \mathcal{K} \backslash\{k\}}\left[\mathbf{w}_{j}\right]^{2}\right\rangle \\
& \leq\left\langle\boldsymbol{X}_{k}, \sum_{j \in \mathcal{K} \backslash\{k\}} \mathbf{Y}_{j}\right\rangle
\end{aligned}
$$

for the Hermitian symmetric matrix variables $\boldsymbol{X}_{k}, k \in \mathcal{K}$ and $\mathbf{Y}_{j}, j \in \mathcal{K}$ of size $M \times M$ satisfying the semi-definite (convex) constraints

$$
\begin{gathered}
\boldsymbol{X}_{k} \succeq\left[\mathcal{H}_{k}^{H}(\boldsymbol{\theta})\right]^{2} \Leftrightarrow\left[\begin{array}{cc}
\boldsymbol{X}_{k} & \mathcal{H}_{k}^{H}(\boldsymbol{\theta}) \\
\mathcal{H}_{k}(\boldsymbol{\theta}) & 1
\end{array}\right] \succeq 0, \\
\mathbf{Y}_{j} \succeq\left[\mathbf{w}_{j}\right]^{2}, j \in \mathcal{K} \Leftrightarrow\left[\begin{array}{cc}
\mathbf{Y}_{j} & \mathbf{w}_{j} \\
\mathbf{w}_{j}^{H} & 1
\end{array}\right] \succeq 0 .
\end{gathered}
$$

For $X_{k}^{(\kappa)}(t) \triangleq\left[\mathcal{H}_{k}^{H}\left(\theta^{(\kappa)}\right)\right]^{2}+t I_{M}$, and $Y_{k}^{(\kappa)}(t)=\left[w_{k}^{(\kappa)}\right]^{2}+$ $t I_{M}$, while $Y_{\backslash k}^{(\kappa)}(t) \triangleq \sum_{j \in \mathcal{K} \backslash\{k\}}\left[w_{j}^{(\kappa)}\right]^{2}+t I_{M}$, for $k \in \mathcal{K}$, using the inequality (78) in the appendix A yields (26) on the top of this page, for $\boldsymbol{X} \triangleq\left\{\boldsymbol{X}_{k}, k \in \mathcal{K}\right\}$ and $\mathbf{Y} \triangleq\left\{\mathbf{Y}_{j}, j \in\right.$ $\mathcal{K}\}$, and $\epsilon>0$.

Next, in the appendix B, we show that the nonconvex constraint

$$
\left|\mathcal{H}_{k}(\boldsymbol{\theta}) \mathbf{w}_{k}\right|^{2} \geq \mathbf{z}_{k},
$$


is innerly approximated by the semi-definite constraint (27) for $\eta>0$, i.e. each feasible point for (27) on the top of the previous page, is also feasible for (36).

It follows from (32), (26) and (27) that

$$
\begin{aligned}
\rho_{k}(\boldsymbol{\theta}, \mathbf{w}) & \geq \rho_{k}^{(\kappa)}\left(\boldsymbol{\theta}, \mathbf{w}, \mathbf{z}_{k}, \boldsymbol{X}_{k}, \mathbf{Y}\right) \\
& \triangleq a_{k}^{(\kappa)}-\frac{b_{k}^{(\kappa)}}{\mathbf{z}_{k}}-c^{(\kappa)} g_{k}^{(\kappa)}\left(\mathbf{w}, \boldsymbol{X}_{k}, \mathbf{Y}\right)
\end{aligned}
$$

for the scalar variable $\mathbf{z}_{k}$ satisfying the semi-definite constraint (27) and the linear constraint

$$
\mathbf{z}_{k}>0 \text {. }
$$

For $g_{p}^{(\kappa)}(\boldsymbol{\theta}, \mathbf{w}, \mathbf{z}, \boldsymbol{X}, \mathbf{Y}) \triangleq \min _{k=1, \ldots, K} \rho_{k}^{(\kappa)}\left(\boldsymbol{\theta}, \mathbf{w}, \mathbf{z}_{k}, b X_{k}\right.$, $\mathbf{Y})+\mu\left(\frac{1}{N}-\iota^{(\kappa)}(\boldsymbol{\theta})\right)$, at the $\kappa$-th iteration we solve the following convex problem of computational complexity $\mathcal{O}\left(\left(2 K M^{2}+K M+K+N\right)^{3}(4 K+N+2)\right)[43$, p. 4] to generate $\left(\theta^{(\kappa+1)}, w^{(\kappa+1)}, z^{(\kappa+1)}, X^{(\kappa+1)}, Y^{(\kappa+1)}\right)$ :

$$
\begin{gathered}
\max _{\boldsymbol{\theta}, \mathbf{w}, \mathbf{z}, \boldsymbol{X}, \mathbf{Y}} g_{p}^{(\kappa)}(\boldsymbol{\theta}, \mathbf{w}, \mathbf{z}, \boldsymbol{X}, \mathbf{Y}) \\
\text { s.t. } \quad(8 b),(10),(29),(34),(35),(38),(27) .
\end{gathered}
$$

For $z_{k}^{(\kappa)}=\left[w_{k}^{(\kappa)}\right]^{2}$ and $z^{(\kappa)}=\left\{z_{k}^{(\kappa)}, k \in \mathcal{K}\right\}$, it is true that

$$
\begin{gathered}
g_{p}^{(\kappa)}\left(\theta^{(\kappa+1)}, w^{(\kappa+1)}, z^{(\kappa+1)}, X^{(\kappa+1)}, Y^{(\kappa+1)}\right) \\
g_{p}^{(\kappa)}\left(\theta^{(\kappa)}, w^{(\kappa)}, z^{(\kappa)}, X^{(\kappa)}, Y^{(\kappa)}\right)
\end{gathered}
$$

because $\quad\left(\theta^{(\kappa+1)}, w^{(\kappa+1)}, z^{(\kappa+1)}, X^{(\kappa+1)}, Y^{(\kappa+1)}\right) \quad$ and $\left(\theta^{(\kappa)}, w^{(\kappa)}, z^{(\kappa)}, X^{(\kappa)}, Y^{(\kappa)}\right)$ are respectively the optimal solution and a feasible point for (39). Also, under (29), (34), (35), (38), (27),

$$
g(\boldsymbol{\theta}, \mathbf{w}) \geq g_{p}^{(\kappa)}(\boldsymbol{\theta}, \mathbf{w}, \mathbf{z}, \boldsymbol{X}, \mathbf{Y}),
$$

and

$$
g\left(\theta^{(\kappa)}, w^{(\kappa)}\right)=g_{p}^{(\kappa)}\left(\theta^{(\kappa)}, w^{(\kappa)}, z^{(\kappa)}, X^{(\kappa)}, Y^{(\kappa)}\right) .
$$

Therefore, like (21), it is easy to show (31) but the sequence $\left\{\left(\theta^{(\kappa)}, w^{(\kappa)}\right)\right\}$ of improved feasible points for the nonconvex problem (7) converges at least to a locally optimal solution of (7) $[35]$.

\section{Initialization and penalty parameter}

We address the following optimization problem

$$
\max _{\boldsymbol{\theta}, \mathbf{w}} f(\boldsymbol{\theta}, \mathbf{w}) \quad \text { s.t. } \quad(10),(8 b)
$$

by Algorithm 1, which is based on the above described alternating descent iterations.

Suppose that $\left(w^{(0)}, \theta^{(0)}\right)$ is the found solution of (40) with the optimal value $\gamma^{(0)}$. Then determine $\mu$ by

$$
\mu=\frac{\gamma^{(0)}}{\frac{1}{\sum_{n=1}^{N}\left|\theta_{n}^{(0)}\right|^{2}}-\frac{1}{N}} .
$$

to make the values of the objective function and penalty term in (20) of similar magnitudes [44].

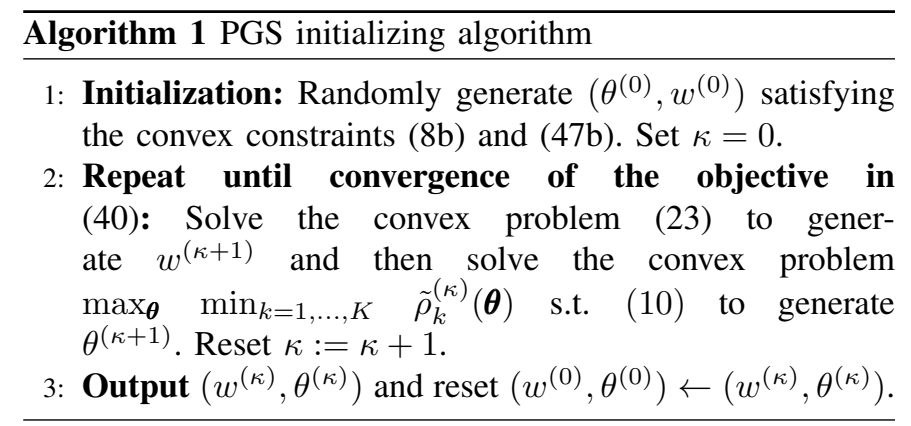

\section{Two-phase Algorithm}

Observe from (23) and (30) for the proposed alternating descent procedure and (39) for the proposed path-following procedure that the latter is much more computationally costly than the latter. Therefore, we propose Algorithm 2 to exploit the computational efficiency of the alternating descent procedure and the solution optimality of the path-following procedure.

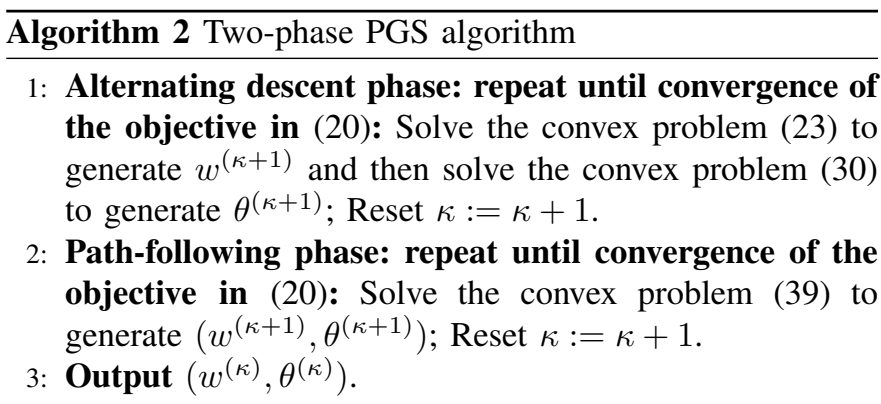

\section{IMPROPER GAUSSIAN SIGNALING}

In (3), the proper Gaussian sources $s_{k}$ are linearly beamformed by the beamformer $\mathbf{w}_{k}$ so the transmit signal $x$ is proper Gaussian too. In this section, the proper Gaussian sources $s_{k}$ are widely linearly beamformed by a pair of two beamformers $\mathbf{w}_{1, k} \in \mathbb{C}^{M}$ and $\mathbf{w}_{2, k} \in \mathbb{C}^{M}$ as [31]

$$
\left[\begin{array}{ll}
\mathbf{w}_{1, k} & \mathbf{w}_{2, k}
\end{array}\right]\left[\begin{array}{c}
s_{k} \\
s_{k}^{*}
\end{array}\right],
$$

making the transmit signal

$$
x=\sum_{k=1}^{K}\left(\mathbf{w}_{1, k} s_{k}+\mathbf{w}_{2, k} s_{k}^{*}\right),
$$

improper Gaussian. The equation (1) is written by

$$
y_{k}=\mathcal{H}_{k}(\boldsymbol{\theta}) \sum_{k=1}^{K}\left(\mathbf{w}_{1, k} s_{k}+\mathbf{w}_{2, k} s_{k}^{*}\right)+n_{k} .
$$

Write the augmented equation for (44) as

$$
\begin{aligned}
{\left[\begin{array}{l}
y_{k} \\
y_{k}^{*}
\end{array}\right] } & =\left[\begin{array}{cc}
\mathcal{H}(\boldsymbol{\theta}) & 0 \\
0 & \mathcal{H}^{*}(\boldsymbol{\theta})
\end{array}\right] \sum_{k=1}^{K}\left[\begin{array}{ll}
\mathbf{w}_{1, k} & \mathbf{w}_{2, k} \\
\mathbf{w}_{2, k}^{*} & \mathbf{w}_{1, k}^{*}
\end{array}\right]\left[\begin{array}{l}
s_{k} \\
s_{k}^{*}
\end{array}\right]+\left[\begin{array}{l}
n_{k} \\
n_{k}^{*}
\end{array}\right] \\
& =\Lambda_{k}(\boldsymbol{\theta}) \sum_{k=1}^{K} \mathbf{W}_{k} \bar{s}_{k}+\bar{n}_{k},
\end{aligned}
$$


for

$$
\Lambda_{k}(\boldsymbol{\theta}) \triangleq\left[\begin{array}{cc}
\mathcal{H}_{k}(\boldsymbol{\theta}) & 0 \\
0 & \mathcal{H}_{k}^{*}(\boldsymbol{\theta})
\end{array}\right] \in \mathbb{C}^{2 \times(2 M)},
$$

and

$$
\mathbf{W}_{k} \triangleq\left[\begin{array}{ll}
\mathbf{w}_{1, k} & \mathbf{w}_{2, k} \\
\mathbf{w}_{2, k}^{*} & \mathbf{w}_{1, k}^{*}
\end{array}\right] \in \mathbb{C}^{2 M \times 2},
$$

which are linear mappings, and

$$
\bar{s}_{k} \triangleq\left[\begin{array}{l}
s_{k} \\
s_{k}^{*}
\end{array}\right] \in \mathbb{C}^{2}, \bar{n}_{k} \triangleq\left[\begin{array}{l}
n_{k} \\
n_{k}^{*}
\end{array}\right] \in \mathbb{C}^{2} .
$$

For $\mathbf{w} \triangleq\left\{\left(\mathbf{w}_{1, k}, \mathbf{w}_{2, k}\right) k \in \mathcal{K}\right\}$, the rate at UE $k$ is calculated by $(1 / 2) r_{k}(\boldsymbol{\theta}, \mathbf{w})$ [45] with

$r_{k}(\boldsymbol{\theta}, \mathbf{w})=\ln \left|I_{2}+\left[\Lambda_{k}(\boldsymbol{\theta}) \mathbf{W}_{k}\right]^{2}\left(\sum_{j \in \mathcal{K} \backslash\{k\}}\left[\Lambda_{k}(\boldsymbol{\theta}) \mathbf{W}_{j}\right]^{2}+\sigma I_{2}\right)^{-1}\right|$

For the particular class $\mathbf{w}_{2, k} \equiv 0$, i.e. $x$ in (43) is proper Gaussian, a straight calculation yields

$$
\begin{aligned}
& r_{k}(\boldsymbol{\theta}, \mathbf{w})= \\
& 2 \ln \left(1+\left|\mathcal{H}_{k}(\boldsymbol{\theta}) \mathbf{w}_{1, k}\right|^{2}\left(\sum_{j \in \mathcal{K} \backslash\{k\}}\left|\mathcal{H}_{k}(\boldsymbol{\theta}) \mathbf{w}_{1, j}\right|^{2}+\sigma\right)^{-1}\right),
\end{aligned}
$$

so $(1 / 2) r_{k}(\boldsymbol{\theta}, \mathbf{w})$ is the known PGS rate (7).

Given a power budget $P$, the max-min rate optimization problem under IGS is thus formulated as

$$
\begin{array}{r}
\max _{\boldsymbol{\theta}, \mathbf{w}} \min _{k=1, \ldots, K} \frac{1}{2} r_{k}(\boldsymbol{\theta}, \mathbf{w}) \quad \text { s.t. } \quad(2), \\
\sum_{k=1}^{K}\left(\left\|\mathbf{w}_{1, k}\right\|^{2}+\left\|\mathbf{w}_{2, k}\right\|^{2}\right) \leq P,
\end{array}
$$

which is equivalent to

$$
\max _{\boldsymbol{\theta}, \mathbf{w}} \Phi(\boldsymbol{\theta}, \mathbf{w}) \triangleq \min _{k=1, \ldots, K} r_{k}(\boldsymbol{\theta}, \mathbf{w}) \quad \text { s.t. } \quad(2),(47 b) .
$$

Like (20), we address (48) via its exact penalized problem

$$
\max _{\boldsymbol{\theta}, \mathbf{w}} \Psi(\boldsymbol{\theta}, \mathbf{w}) \triangleq\left[\Phi(\boldsymbol{\theta}, \mathbf{w})+\mu\left(\frac{1}{N}-\frac{1}{\sum_{n=1}^{N}\left|\theta_{n}\right|^{2}}\right)\right]
$$$$
\text { s.t. }(10),(47 b) \text {, }
$$

where $\mu>0$ is the penalty parameter. Unlike its PGS counterpart (20), which involves a single beamformer for each user, the problem (49) involves a pairs of correlated beamformers $\mathbf{w}_{1, k}$ and $\mathbf{w}_{2, k}$. More importantly, the problem (49) is a $\log$ determinant function optimization and thus is much more computationally challenging than its PGS counterpart (20) of fractional function optimization. Particularly, the Algorithms 1 and 2 for PGS cannot be extended to the case of IGS. Nevertheless, we are still able to propose alternating descent and path-following iterations tailored for its computation.

Let $\left(w^{(\kappa)}, \theta^{(\kappa)}\right)$ be the feasible point for (48) that is found from the $(\kappa-1)$-th round.

\section{A. Alternating descent round}

1) Beamforming descent iteration: We seek $w^{(\kappa+1)}$ such that

$$
\Phi\left(\theta^{(\kappa)}, w^{(\kappa+1)}\right)>\Phi\left(\theta^{(\kappa)}, w^{(\kappa)}\right) .
$$

By using the inequality (74) in the appendix A, we obtain a concave quadratic lower bounding function approximation of $r_{k}\left(\theta^{(\kappa)}, \mathbf{w}\right)$ as

$$
\begin{aligned}
r_{k}\left(\theta^{(\kappa)}, \mathbf{w}\right) \geq & r_{k}^{(\kappa)}(\mathbf{w}) \\
\triangleq & a_{k}^{(\kappa)}+2 \Re\left\{\left\langle B_{k}^{(\kappa)} \mathbf{W}_{k}\right\rangle\right\} \\
& -\left\langle C_{k}^{(\kappa)}, \sum_{j \in \mathcal{K}}\left[\Lambda_{k}\left(\theta^{(\kappa)}\right) \mathbf{W}_{j}\right]^{2}\right\rangle,
\end{aligned}
$$

with

$$
\begin{aligned}
& a_{k}^{(\kappa)} \triangleq r_{k}\left(\theta^{(\kappa)}, w^{(\kappa)}\right)-\left\langle\left[\Lambda_{k}\left(\theta^{(\kappa)}\right) W_{k}^{(\kappa)}\right]^{2}\left(Y_{k}^{(\kappa)}\right)^{-1}\right\rangle-\sigma\left\langle C_{k}^{(\kappa)}\right\rangle, \\
& B_{k}^{(\kappa)} \triangleq\left(W_{k}^{(\kappa)}\right)^{H}\left(\Lambda_{k}\left(\theta^{(\kappa)}\right)\right)^{H}\left(Y_{k}^{(\kappa)}\right)^{-1} \Lambda_{k}\left(\theta^{(\kappa)}\right) \\
& 0 \prec C_{k}^{(\kappa)} \triangleq\left(Y_{k}^{(\kappa)}\right)^{-1}-\left(Y_{k}^{(\kappa)}+\left[\Lambda_{k}\left(\theta^{(\kappa)}\right) W_{k}^{(\kappa)}\right]^{2}\right)^{-1} \text {, } \\
& 0 \prec Y_{k}^{(\kappa)} \triangleq \sum_{j \in \mathcal{K} \backslash\{k\}}\left[\Lambda_{k}\left(\theta^{(\kappa)}\right) W_{j}^{(\kappa)}\right]^{2}+\sigma I_{2} .
\end{aligned}
$$

We solve the following convex problem of computational complexity $\mathcal{O}\left((2 M K)^{3}\right)[43$, p. 4$]$ at the $\kappa$-th iteration to generate $w^{(\kappa+1)}$ :

$$
\max _{\mathbf{w}} \min _{k=1, \ldots, K} r_{k}^{(\kappa)}(\mathbf{w}) \quad \text { s.t. } \quad(47 b),
$$

which like (23) gives (50) as far as $w^{(\kappa+1)} \neq w^{(\kappa)}$.

2) Phase descent iteration: We seek $w^{(\kappa+1)}$ such that

$$
\Psi\left(\theta^{(\kappa+1)}, w^{(\kappa+1)}\right)>\Psi\left(\theta^{(\kappa)}, w^{(\kappa+1)}\right) .
$$

By using the inequality (74) in the appendix A, we obtain a concave quadratic lower bounding function approximation of $r_{k}\left(\boldsymbol{\theta}, w^{(\kappa+1)}\right)$ as

$$
\begin{aligned}
r_{k}\left(\boldsymbol{\theta}, w^{(\kappa+1)}\right) \geq & \tilde{r}_{k}^{(\kappa)}(\boldsymbol{\theta}) \\
\triangleq & \tilde{a}_{k}^{(\kappa)}+2 \Re\left\{\left\langle\tilde{B}_{k}^{(\kappa)} \Lambda_{k}(\boldsymbol{\theta}) W_{k}^{(\kappa+1)}\right\rangle\right\} \\
& -\left\langle\tilde{C}_{k}^{(\kappa)}, \sum_{j \in \mathcal{K}}\left[\Lambda_{k}(\boldsymbol{\theta}) W_{j}^{(\kappa+1)}\right]^{2}\right\rangle
\end{aligned}
$$

with

$$
\begin{aligned}
& \tilde{a}^{(\kappa)} \triangleq r_{k}\left(\theta^{(\kappa)}, w^{(\kappa+1)}\right) \\
& -\left\langle\left[\Lambda_{k}\left(\theta^{(\kappa)}\right) W_{k}^{(\kappa+1)}\right]^{2}\left(Y_{k}^{(\kappa+1)}\right)^{-1}\right\rangle-\sigma\left\langle\tilde{C}_{k}^{(\kappa)}\right\rangle, \\
& \tilde{B}_{k}^{(\kappa)} \triangleq\left(W_{k}^{(\kappa+1)}\right)^{H}\left(\Lambda_{k}\left(\theta^{(\kappa)}\right)\right)^{H}\left(Y_{k}^{(\kappa+1)}\right)^{-1}, \\
& 0 \prec \tilde{C}_{k}^{(\kappa)} \triangleq\left(Y_{k}^{(\kappa+1)}\right)^{-1}-\left(Y_{k}^{(\kappa+1)}+\left[\Lambda_{k}\left(\theta^{(\kappa)}\right) W_{k}^{(\kappa+1)}\right]^{2}\right)^{-1} \text {, } \\
& 0 \prec Y_{k}^{(\kappa+1)} \triangleq \sum_{j \in \mathcal{K} \backslash\{k\}}\left[\Lambda_{k}\left(\theta^{(\kappa)}\right) W_{j}^{(\kappa+1)}\right]^{2}+\sigma I_{2} \text {. }
\end{aligned}
$$

Accordingly, we solve the following convex problem of computational complexity $\mathcal{O}\left(N^{3}(N+1)\right)$ [43, p. 4] at the $\kappa$-th iteration to generate $\theta^{(\kappa+1)}$ :

$\max _{\boldsymbol{\theta}}\left[\min _{k=1, \ldots, K} \tilde{r}_{k}^{(\kappa)}(\boldsymbol{\theta})+\mu\left(\frac{1}{N}-\iota^{(\kappa)}(\boldsymbol{\theta})\right)\right] \quad$ s.t. $\quad(10),(29)$,

where $\iota^{(\kappa)}(\boldsymbol{\theta})$ is recalled from (28).

Like (24), we can easily show (53) as far as $\theta^{(\kappa+1)} \neq \theta^{(\kappa)}$. 


$$
\begin{aligned}
\text { RHS of (58) }= & a_{k}^{(\kappa)}-\left\langle\boldsymbol{X}_{1 k}+\epsilon I_{2 M}, \mathbf{Y}_{k}+\epsilon I_{2 M}\right\rangle-\left\langle\boldsymbol{X}_{2, k}+\epsilon I_{2 M}, \sum_{j \in \mathcal{K} \backslash\{k\}} \mathbf{Y}_{j}+\epsilon I_{2 M}\right\rangle \\
& +\epsilon \sum_{j \in \mathcal{K}}\left\langle\mathbf{Y}_{j}\right\rangle+\epsilon\left\langle\boldsymbol{X}_{1 k}+\boldsymbol{X}_{2 k}\right\rangle+2 M \epsilon^{2} \\
\geq & a_{k}^{(\kappa)}-\frac{1}{2}\left\|\left(X_{1, k}^{(\kappa)}(\epsilon)\right)^{-1 / 2}\left(\boldsymbol{X}_{1 k}+\epsilon I_{2 M}\right)\left(Y_{k}^{(\kappa)}(\epsilon)\right)^{1 / 2}\right\|^{2} \\
& -\frac{1}{2}\left\|\left(X_{1, k}^{(\kappa)}(\epsilon)\right)^{1 / 2}\left(\mathbf{Y}_{k}+\epsilon I_{2 M}\right)\left(Y_{k}^{(\kappa)}(\epsilon)\right)^{-1 / 2}\right\|^{2} \\
& -\frac{1}{2}\left\|\left(X_{2, k}^{(\kappa)}(\epsilon)\right)^{-1 / 2}\left(\boldsymbol{X}_{2, k}+\epsilon I_{2 M}\right)\left(Y_{\backslash k}^{(\kappa)}(\epsilon)\right)^{1 / 2}\right\|^{2} \\
& -\frac{1}{2}\left\|\left(X_{2, k}^{(\kappa)}(\epsilon)\right)^{1 / 2}\left(\sum_{j \in \mathcal{K} \backslash\{k\}} \mathbf{Y}_{j}+\epsilon I_{2 M}\right)\left(Y_{\backslash k}^{(\kappa)}(\epsilon)\right)^{-1 / 2}\right\|^{2} \\
& +\epsilon \sum_{j \in \mathcal{K}}\left\langle\mathbf{Y}_{j}\right\rangle+\epsilon\left\langle\boldsymbol{X}_{1 k}+\boldsymbol{X}_{2 k}\right\rangle+2 M \epsilon^{2} \\
\triangleq & \varphi_{k}^{(\kappa)}\left(\boldsymbol{\theta}, \boldsymbol{X}_{1, k}, \boldsymbol{X}_{2, k}, \mathbf{Y}_{k},\right.
\end{aligned}
$$

$$
\begin{aligned}
& {\left[\begin{array}{cc}
\Lambda_{k}\left(\theta^{(\kappa)}\right) Y_{k}^{(\kappa)}(\eta) \Lambda_{k}^{H}(\boldsymbol{\theta})+(*)^{H} & * \\
Y_{k}^{(\kappa)}(\eta) \Lambda_{k}^{H}\left(\theta^{(\kappa)}\right) & {\left[W_{k}^{(\kappa)}\left(\mathbf{W}_{k}\right)^{H}+(*)^{H}\right]+\eta I_{2 M}}
\end{array}\right] \succeq} \\
& {\left[\begin{array}{cc}
\mathbf{Z}_{k}+\eta \boldsymbol{Q}_{k} & 0_{2 \times(2 M)} \\
0_{(2 M) \times 2} & {\left[W_{k}^{(\kappa)}\right]^{2}}
\end{array}\right]} \\
& {\left[\begin{array}{cc}
\mathbf{Z}_{k} & \Lambda_{k}(\boldsymbol{\theta}) \\
\Lambda_{k}^{H}(\boldsymbol{\theta}) & I_{2 M}
\end{array}\right] \succeq 0}
\end{aligned}
$$

\section{B. Path-following round}

Decompose $r_{k}(\boldsymbol{\theta}, \mathbf{w})=\psi_{k}(\boldsymbol{\theta}, \mathbf{w})+\varphi_{k}(\boldsymbol{\theta}, \mathbf{w})$, for $\psi_{k}(\boldsymbol{\theta}, \mathbf{w}) \triangleq \ln \left|\left[\Lambda_{k}(\boldsymbol{\theta}) \mathbf{W}_{k}\right]^{2}\right|$, and

$$
\begin{aligned}
& \varphi_{k}(\boldsymbol{\theta}, \mathbf{w}) \triangleq \\
& \quad \ln \left|\left[\Lambda_{k}(\boldsymbol{\theta}) \mathbf{W}_{k}\right]^{-2}+\left(\sum_{j \in \mathcal{K} \backslash\{k\}}\left[\Lambda_{k}(\boldsymbol{\theta}) \mathbf{W}_{j}\right]^{2}+\sigma I_{2}\right)^{-1}\right| .
\end{aligned}
$$

Using the inequalities (75) in the appendix A yields

$$
\begin{aligned}
\varphi_{k}(\boldsymbol{\theta}, \mathbf{w}) \geq & a_{k}^{(\kappa)}-\left\langle B_{k}^{(\kappa)},\left[\Lambda_{k}(\boldsymbol{\theta}) \mathbf{W}_{k}\right]^{2}\right\rangle \\
& -\left\langle C_{k}^{(\kappa)}, \sum_{j \in \mathcal{K} \backslash\{k\}}\left[\Lambda_{k}(\boldsymbol{\theta}) \mathbf{W}_{j}\right]^{2}\right\rangle \\
= & a_{k}^{(\kappa)}-\left\langle\Lambda_{k}^{H}(\boldsymbol{\theta}) B_{k}^{(\kappa)} \Lambda_{k}(\boldsymbol{\theta}),\left[\mathbf{W}_{k}\right]^{2}\right\rangle \\
& -\left\langle\Lambda_{k}^{H}(\boldsymbol{\theta}) C_{k}^{(\kappa)} \Lambda_{k}(\boldsymbol{\theta}), \sum_{j \in \mathcal{K} \backslash\{k\}}\left[\mathbf{W}_{j}\right]^{2}\right\rangle \\
\geq & a_{k}^{(\kappa)}-\left\langle\boldsymbol{X}_{1 k}, \mathbf{Y}_{k}\right\rangle-\left\langle\boldsymbol{X}_{2, k}, \sum_{j \in \mathcal{K} \backslash\{k\}} \mathbf{Y}_{j}\right\rangle(58)
\end{aligned}
$$

for the newly introduced Hermitian symmetric matrix variables $\boldsymbol{X}_{1, k}$ and $\boldsymbol{X}_{2, k}, k \in \mathcal{K}$ and $\mathbf{Y}_{j}, j \in \mathcal{K}$ of size $(2 M) \times(2 M)$ satisfying the semi-definite constraints

$$
\begin{aligned}
\boldsymbol{X}_{1, k} & \succeq \Lambda_{k}^{H}(\boldsymbol{\theta}) B_{k}^{(\kappa)} \Lambda_{k}(\boldsymbol{\theta}) \\
& \Leftrightarrow\left[\begin{array}{cc}
\boldsymbol{X}_{1, k} & \Lambda_{k}^{H}(\boldsymbol{\theta})\left(B_{k}^{(\kappa)}\right)^{1 / 2} \\
\left(B_{k}^{(\kappa)}\right)^{1 / 2} \Lambda_{k}(\boldsymbol{\theta}) & I_{2}
\end{array}\right] \succeq 0,
\end{aligned}
$$

and

$$
\begin{aligned}
\boldsymbol{X}_{2, k} & \succeq \quad \Lambda_{k}^{H}(\boldsymbol{\theta}) C_{k}^{(\kappa)} \Lambda_{k}(\boldsymbol{\theta}) \\
& \Leftrightarrow\left[\begin{array}{cc}
\boldsymbol{X}_{2, k} & \Lambda_{k}^{H}(\boldsymbol{\theta})\left(C_{k}^{(\kappa)}\right)^{1 / 2} \\
\left(C_{k}^{(\kappa)}\right)^{1 / 2} \Lambda_{k}(\boldsymbol{\theta}) & I_{2}
\end{array}\right] \succeq 0, \\
\text { and } & \mathbf{Y}_{j} \succeq\left[\mathbf{W}_{j}\right]^{2}, \Leftrightarrow\left[\begin{array}{cc}
\mathbf{Y}_{j} & \mathbf{W}_{j} \\
\mathbf{W}_{j}^{H} & I_{2}
\end{array}\right] \succeq 0,
\end{aligned}
$$

under the definitions

$$
\begin{aligned}
a_{k}^{(\kappa)} \triangleq \varphi_{k}\left(\theta^{(\kappa)}, w^{(\kappa)}\right)+2-\sigma\left\langle C_{k j}^{(\kappa)}\right\rangle \quad(j \neq k), & \\
0 \preceq B_{k}^{(\kappa)} & \triangleq\left(\left[\Lambda_{k}\left(\theta^{(\kappa)}\right) W_{k}^{(\kappa)}\right]^{2}\right)^{-1} \\
& -\left(\sum_{j \in \mathcal{K}}\left[\Lambda_{k}\left(\theta^{(\kappa)}\right) W_{j}^{(\kappa)}\right]^{2}+\sigma I_{2}\right)^{-1}, \\
0 \preceq C_{k}^{(\kappa)} \triangleq & \left(\sum_{j \in \mathcal{K} \backslash\{k\}}\left[\Lambda_{k}\left(\theta^{(\kappa)}\right) W_{j}^{(\kappa)}\right]^{2}+\sigma I_{2}\right)^{-1} \\
& -\left(\sum_{j \in \mathcal{K}}\left[\Lambda_{k}\left(\theta^{(\kappa)}\right) W_{j}^{(\kappa)}\right]^{2}+\sigma I_{2}\right)^{-1},
\end{aligned}
$$

and $X_{1, k}^{(\kappa)} \triangleq \Lambda_{k}^{H}\left(\theta^{(\kappa)}\right) B_{k}^{(\kappa)} \Lambda_{k}\left(\theta^{(\kappa)}\right), \quad$ and $\quad X_{2, k}^{(\kappa)} \triangleq$ $\Lambda_{k}^{H}\left(\theta^{(\kappa)}\right) C_{k}^{(\kappa)} \Lambda_{k}\left(\theta^{(\kappa)}\right)$.

Furthermore, using the inequality (78) in the appendix A yields (56) on the top of this page for

$$
\begin{gathered}
X_{i, k}^{(\kappa)}(t) \triangleq X_{i, k}^{(\kappa)}+t I_{2 M}, i \in\{1,2\}, \\
Y_{k}^{(\kappa)}(t) \triangleq\left[W_{k}^{(\kappa)}\right]^{2}+t I_{2 M},
\end{gathered}
$$


and

$$
Y_{\backslash k}^{(\kappa)}(t) \triangleq \sum_{j \in \mathcal{K} \backslash\{k\}}\left[W_{j}^{(\kappa)}\right]^{2}+t I_{2 M}
$$

Next, similarly to (27), the nonconvex constraint

$$
\left[\Lambda_{k}(\boldsymbol{\theta}) \mathbf{W}_{k}\right]^{2} \succeq \mathbf{Z}_{k},
$$

for the newly introduced Hermitian symmetric matrix variable $\mathbf{Z}_{k}$ of size $2 \times$, is innerly approximated by the semi-definite constraint (57) on the top of the previous page for $\eta>0$ and the slack Hermitian symmetric matrix variable $\boldsymbol{Q}_{k}$ of size $2 \times 2$.

The inequality (57) together with the inequality (76) in the appendix A yield

$$
\begin{aligned}
\psi_{k}(\boldsymbol{\theta}, \mathbf{w}) & \geq \psi_{k}^{(\kappa)}\left(\boldsymbol{\theta}, \mathbf{Z}_{k}\right) \\
& \triangleq \psi_{k}\left(\theta^{(\kappa)}, w^{(\kappa)}\right)-\left\langle\left[\Lambda_{k}\left(\theta^{(\kappa)}\right) W_{k}^{(\kappa)}\right]^{2},\left(\mathbf{Z}_{k}\right)^{-1}\right\rangle
\end{aligned}
$$

under the trust region

$$
\mathbf{Z}_{k} \succ 0 .
$$

Thus, at the $\kappa$-th iteration we solve the following convex problem of inner approximation of (49) with computational complexity $\mathcal{O}\left(\left(12 K M^{2}+2 K M+4 K+N\right)^{3}(5 K+N+2)\right)$ $\left[43\right.$, p. 4] to generate $\left(\theta^{(\kappa+1)}, w^{(\kappa+1)}\right)$ :

$$
\begin{array}{r}
\max _{\boldsymbol{\theta}, \mathbf{w}, \boldsymbol{X}, \mathbf{Y}, \mathbf{Z}}\left[\operatorname { m i n } _ { k = 1 , \ldots , K } \left[\varphi_{k}^{(\kappa)}\left(\boldsymbol{\theta}, \boldsymbol{X}_{1, k}, \boldsymbol{X}_{2, k}, \mathbf{Y}\right)\right.\right. \\
\left.\left.+\psi_{k}^{(\kappa)}\left(\boldsymbol{\theta}, \mathbf{Z}_{k}\right)\right]+\mu\left(\frac{1}{N}-\iota^{(\kappa)}(\boldsymbol{\theta})\right)\right]
\end{array}
$$$$
\text { s.t. }(10),(29),(47 b),(59),(60),(61),(57),(64) \text {, }
$$

where $\iota^{(\kappa)}(\boldsymbol{\theta})$ is recalled from (28).

\section{Initialization and penalty parameter}

We use Algorithm 3 for computing

$$
\max _{\boldsymbol{\theta}, \mathbf{w}} \Phi(\boldsymbol{\theta}, \mathbf{w}) \quad \text { s.t. } \quad(47 b),(10)
$$

Suppose that $\left(w^{(0)}, \theta^{(0)}\right)$ is the found solution of (66) with the optimal value $\gamma^{(0)}$. Then determine $\mu$ by (41). It is noteworthy that the optimal solution $\mathbf{w}_{1, k}$ and $\mathbf{w}_{2, k}$ from Algorithm 3 are not the complex conjugate of each other.

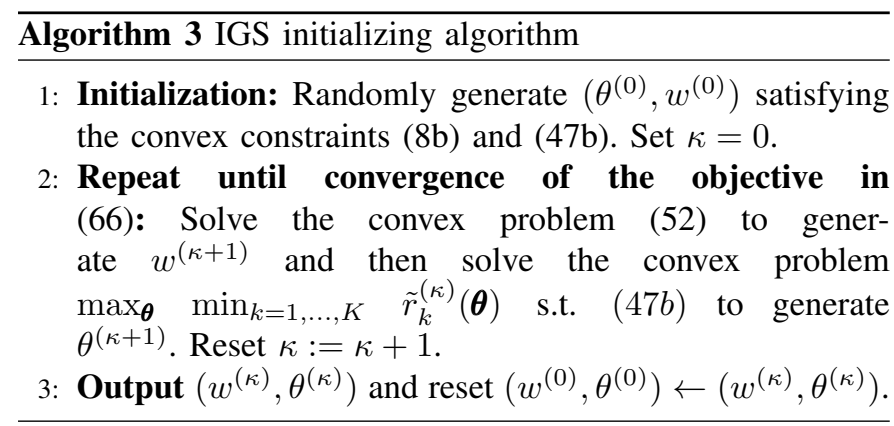

\section{Two-phase Algorithm}

We propose 4, which like Algorithm 2 consists of two phases to exploit the computational efficiency of the alternating descent procedure and the solution optimality of the path-following procedure.

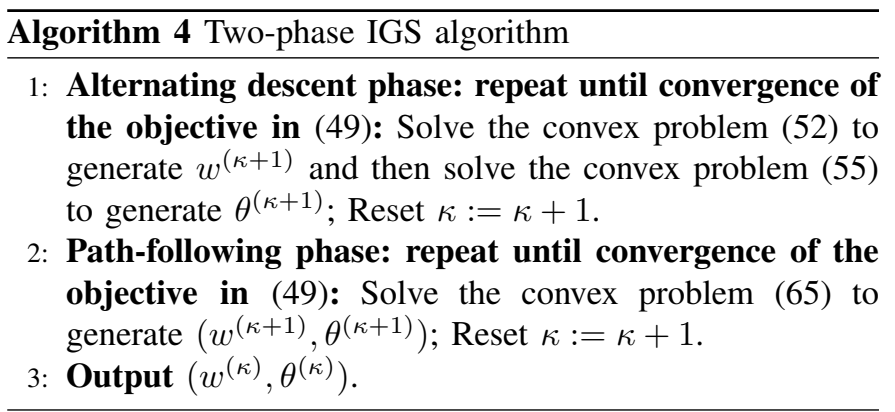

\section{Performance Results}

In this section, we investigate the performance of our proposed algorithms by numerical examples. The large scale fading coefficients, $\beta_{\mathrm{AP}-\mathrm{RIS}}, \beta_{\mathrm{RIS}-\mathrm{k}}$, and $\beta_{\mathrm{AP}-\mathrm{k}}$, in (5), are modeled as [18], [34]

$$
\begin{aligned}
& \beta_{\mathrm{AP}-\mathrm{RIS}}=G_{\mathrm{AP}}+G_{\mathrm{RIS}}-35.9-22 \log _{10}\left(d_{\mathrm{AP}-\mathrm{RIS}}\right)(\text { in } \mathrm{dB}), \\
& \beta_{\mathrm{RIS}-\mathrm{k}}=G_{\mathrm{RIS}}-33.05-30 \log _{10}\left(d_{\mathrm{RIS}-\mathrm{k}}\right)(\text { in } \mathrm{dB}),(67 \mathrm{a}) \\
& \beta_{\mathrm{AP}-\mathrm{k}}=G_{\mathrm{AP}}-33.05-36.7 \log _{10}\left(d_{\mathrm{AP}-\mathrm{k}}\right)(\text { in } \mathrm{dB}),(67 \mathrm{c})
\end{aligned}
$$

where $G_{\mathrm{AP}}=5 \mathrm{dBi}$ and $G_{\mathrm{RIS}}=5 \mathrm{dBi}$ denote the antenna gain of the AP and the gain of the elements of RIS, respectively [18], [34], $d_{\mathrm{AP}-\mathrm{RIS}}, d_{\mathrm{RIS}-\mathrm{k}}$, and $d_{\mathrm{AP}-\mathrm{k}}$ are the distances between the AP and RIS, the RIS and UE $k$, and the AP and UE $k$, respectively. The full-rank AP-to-RIS LoS channel matrix is defined as $\left[H_{A R}\right]_{n, m}=e^{j \pi\left((n-1) \sin \bar{\theta}_{n} \sin \bar{\phi}_{n}+(m-1) \sin \theta_{n} \sin \phi_{n}\right)}$, where $\theta_{n}$ and $\phi_{n}$ are uniformly distributed as $\theta_{n} \sim \mathcal{U}(0, \pi)$ and $\phi_{n} \sim \mathcal{U}(0,2 \pi)$, respectively, and $\bar{\theta}_{n}=\pi-\theta_{n}$ and $\bar{\phi}_{n}=\pi+\phi_{n}$ [18]. The normalized small-scale fading channel $h_{a, k}$ follows Rayleigh distribution while the small-scale fading channel gain $h_{r, k}$ follow Rician distribution with a Rician $\mathrm{K}$-factor of 3. The spatial correlation matrix is given as

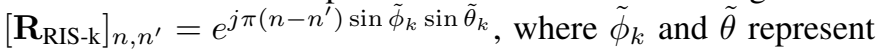
the azimuth and elevation angle for UE $k$, respectively. The noise power is set to $\sigma=-114 \mathrm{dBm}$, i.e., noise power spectral density $=-174 \mathrm{dBm} / \mathrm{Hz}$ and transmission bandwidth $=1$ MHz.

Considering the system model setup in Fig. 1 and let us use $(x, y, z)$ to denote the coordinates (placement) of the AP, RIS and UEs, the AP is deployed at $(40,0,25)$, the RIS is deployed at $(0,60,40)$, and $K=10$ UEs are randomly placed in $120 \mathrm{~m} \times 120 \mathrm{~m}$ right-hand-side of the obstacles and RIS. The following results have been plotted to analyze the performance of our proposed algorithms, where the tolerance level for the convergence of algorithms is set to $10^{-3}$.

- PSG-RIS: This result simulates the performance of PGS algorithm. Particularly, the proposed Alg. 1 is simulated for initialization and proposed Alg. 2 is simulated during optimization phase.

- IGS-RIS: This result simulates the performance of IGS algorithm. Particularly, the proposed Alg. 3 is simulated for initialization and proposed Alg. 4 is simulated during optimization phase.

- PGS-RIS with random $\boldsymbol{\theta}$ : This result simulates the performance of PGS algorithm without phase optimization, i.e., 
it assumes random phase coefficients $\boldsymbol{\theta}$ at the RIS. This result demonstrates the gain achieved by the proposed PGS-RIS algorithm, which assumes joint phase optimization with beamforming design.

- IGS-RIS with random $\theta$ : This result simulates the performance of IGS algorithm by assuming random phase coefficients $\boldsymbol{\theta}$ at the RIS. This result demonstrates the gain achieved by the proposed IGS-RIS algorithm, which assumes joint phase optimization with beamforming design.

- PGS without RIS This result simulates the performance of PGS algorithm in the absence of RIS. This result demonstrates the advantage of deploying RIS.

- PGS without RIS This result simulates the performance of IGS algorithm in the absence of RIS. This result demonstrates the advantage of deploying RIS.

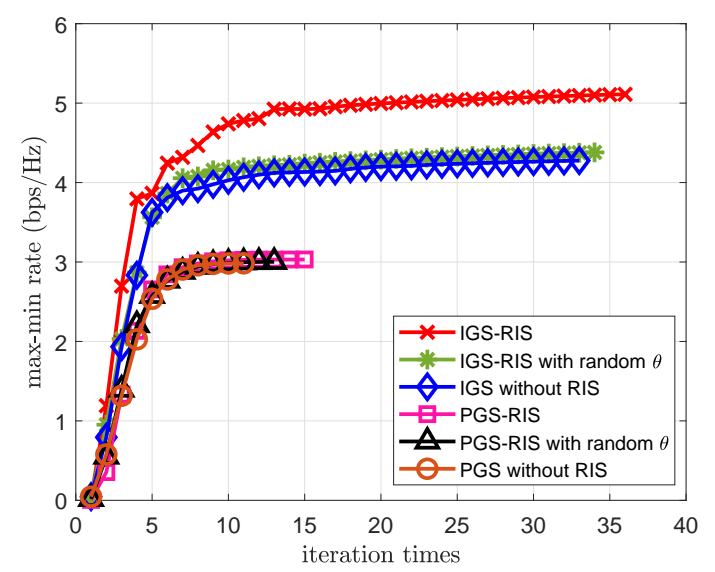

Fig. 2: Convergence with $P=20 \mathrm{dBm}, M=9$ AP-antennas, and $N=100$ RIS elements.

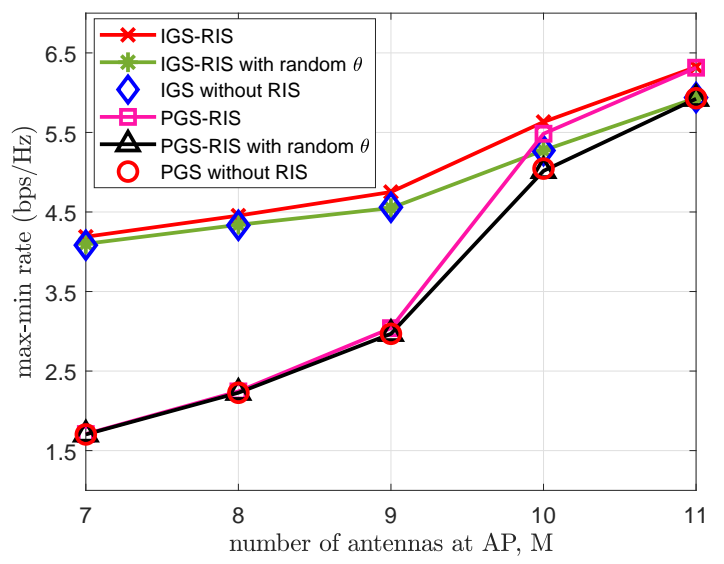

Fig. 3: Achievable max-min rate versus the number of antennas at the AP, $M$, with $P=20 \mathrm{dBm}$ and $N=100$ RIS elements.

Fig. 2 plots the convergence of the proposed algorithms with $P=20 \mathrm{dBm}, M=9 \mathrm{AP}$-antennas, and $N=100$ RIS elements. Fig. 2 assumes the presence of the direct path between the AP and the UEs. It can be seen from Fig. 2 that

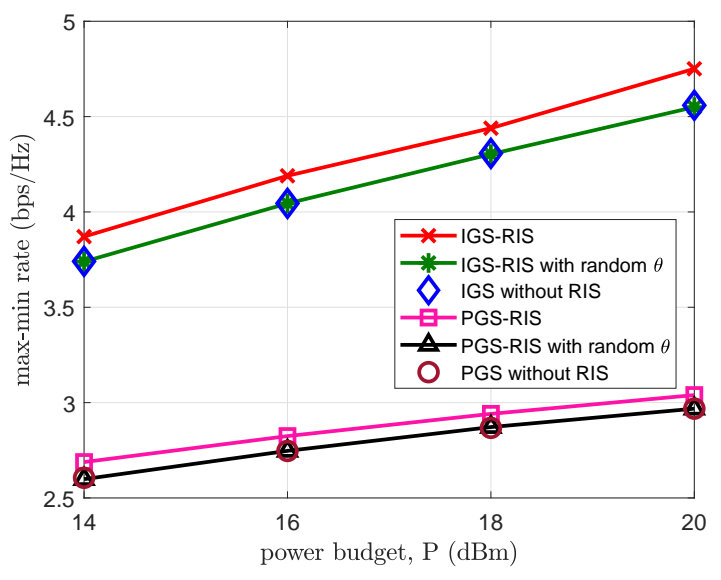

Fig. 4: Achievable max-min rate versus the transmit power budget at the AP, $P$, with $M=9 \mathrm{AP}$-antennas and $N=100$ RIS elements.

all the algorithms converges rapidly within a few iterations (15-30). As expected, the PGS based algorithms converge rapidly than the IGS based algorithms because the latter need to handle more optimization variables. Fig. 3 plots the achievable max-min rate versus the number of antennas at the AP, $M$, with $P=20 \mathrm{dBm}$ and $N=100$ RIS elements. The results have been plotted for the side-range of AP-antennas $M=\{7,8,9,10,11\}$ to consider all three situations; (i) $M<K$, (ii) $M=K$, and (iii) $M>K$, where $K=10$ is the number of UEs as described previously. Fig. 3 shows that the proposed IGS-RIS algorithm clearly outperforms the "IGS without RIS" and "IGS-RIS with random $\theta$ ". The performance margin increases with the increase in $M$. Fig. 3 shows that "IGS without RIS" and "IGS-RIS with random $\theta$ " yield similar performance which provides an important insight that there is no advantage of deploying RIS unless RIS reflection coefficients are optimized. Fig. 3 also plots the performance of the proposed PGS-RIS algorithm which outperforms the "PGS without RIS" and "PGS-RIS with random $\theta$ ". Fig. 3 also shows that the performance of PGS-RIS performance gets closer to that of IGS-RIS for $M>K$, i.e., $M=11$ AP-antennas.

Fig. 4 plots the achievable max-min rate versus the transmit power budget at the $\mathrm{AP}, P$, with $M=9 \mathrm{AP}$-antennas and $N=100$ RIS elements. As expected, the performance of the proposed IGS-RIS and PGS-RIS algorithms improve with the increase in the available power budget. Fig. 4 also shows the performance gain of the proposed IGS-RIS over "IGS without RIS" and "IGS-RIS with random $\theta$ " while the latter two yield similar performance. Similarly, Fig. 4 shows the performance gain of the proposed PGS-RIS over "PGS without RIS" and "PGS-RIS with random $\theta$ ". Fig. 4 clearly shows the advantage of employing IGS over PGS.

Fig. 5 plots the achievable max-min rate versus the number of RIS elements, $N$, with $M=9 \mathrm{AP}$-antennas and $P=20$ dBm. In Fig. 5, $N=0$ implies IGS or PGS without RIS deployment. Fig. 5 shows that only the performance of the proposed algorithm IGS-RIS algorithm improves with the increase in the number of RIS elements. Fig. 5 shows that the proposed 


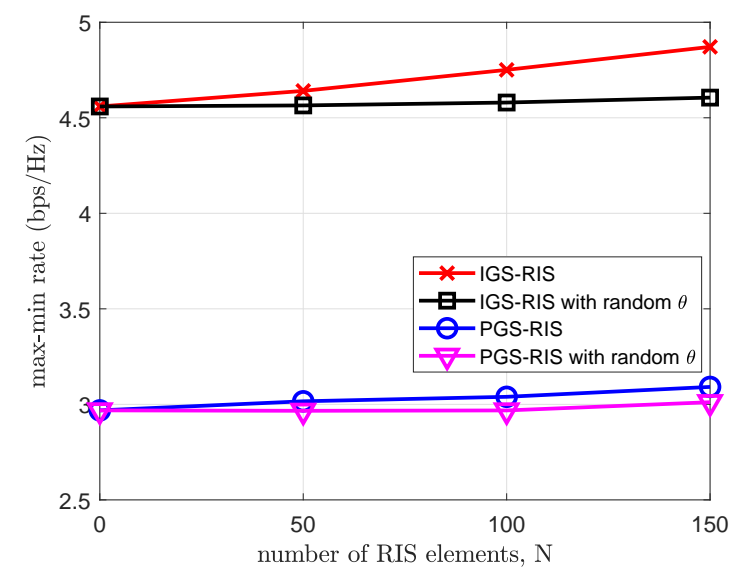

Fig. 5: Achievable max-min rate versus the number of RIS elements, $N$, with $M=9 \mathrm{AP}$-antennas and $P=20 \mathrm{dBm}$.

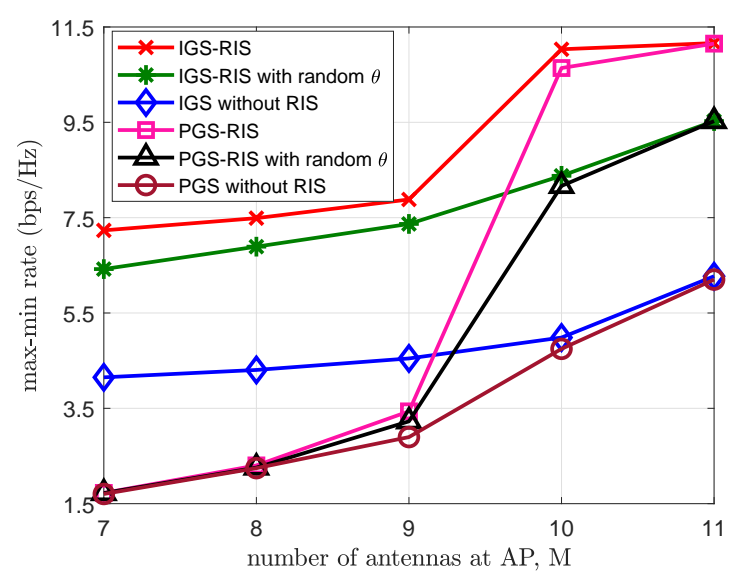

Fig. 6: Under the setup in Remark 1, achievable max-min rate versus the number of antennas at the AP with $P=20 \mathrm{dBm}$ and $N=100$ RIS elements.

IGS-RIS algorithm clearly outperforms the "IGS-RIS with random $\boldsymbol{\theta}$ " and the performance margin increases with the increase in $N$. Similarly, Fig. 5 shows the performance gain of the proposed PGS-RIS over "PGS-RIS with random $\boldsymbol{\theta}$ ". Fig. 5 clearly shows the advantage of employing IGS over PGS.

Remark 1: In this paper, we consider more or less a practical RIS in the diffuse scattering regime with the size of each its meta-surface of the order of the radio wavelength [16]. The product of the two path-losses in the AP-RIS-UE reflected link (see (1)) attenuates it very much (see [34] for analysis in details). Both PGS-RIS and IGS-RIS can achieve much more significant gains in the anomalous reflection regime with the size of each RIS meta surface of ten times larger than the radio wavelength [16]. The path-loss of the reflected path then follows the model which is inversely proportional to sum of the two distances of AP-RIS and RIS-AP links [8], making the AP-RIS-UE reflected link in much better condition. For illustrative purpose, Fig. 6 plots the achievable max-min rate vs the number of antennas at $\mathrm{AP}$ for $\beta_{\mathrm{AP}-\mathrm{RIS}} \beta_{\mathrm{RIS}-\mathrm{k}}$ in (1) modelled by $\beta_{\mathrm{AP}-\mathrm{RIS}-\mathrm{k}}=G_{\mathrm{AP}}-33.05-30 \log _{10}\left(d_{\mathrm{AP}-\mathrm{RIS}}+d_{\mathrm{RIS}-\mathrm{k}}\right)($ in $\mathrm{dB})$.

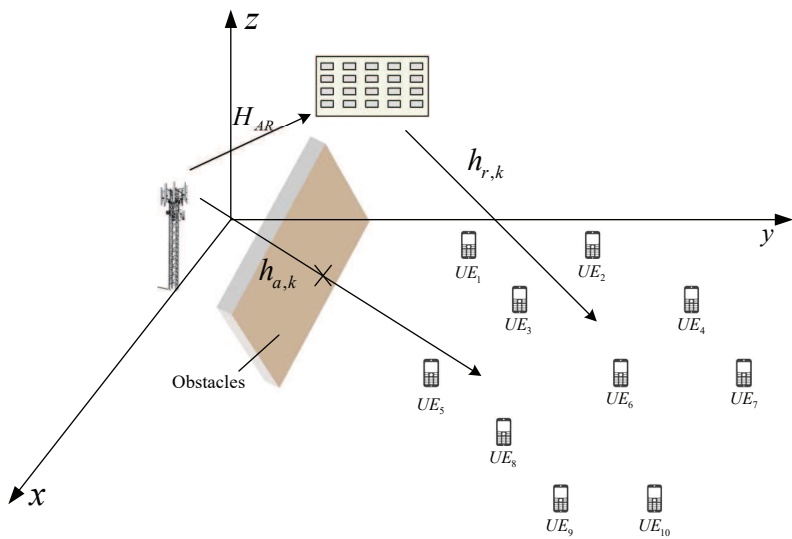

Fig. 7: A simulation setup with the blockage of the direct path bewteen the AP and the UEs. The results for this setup are shown in Figs. 8-10.

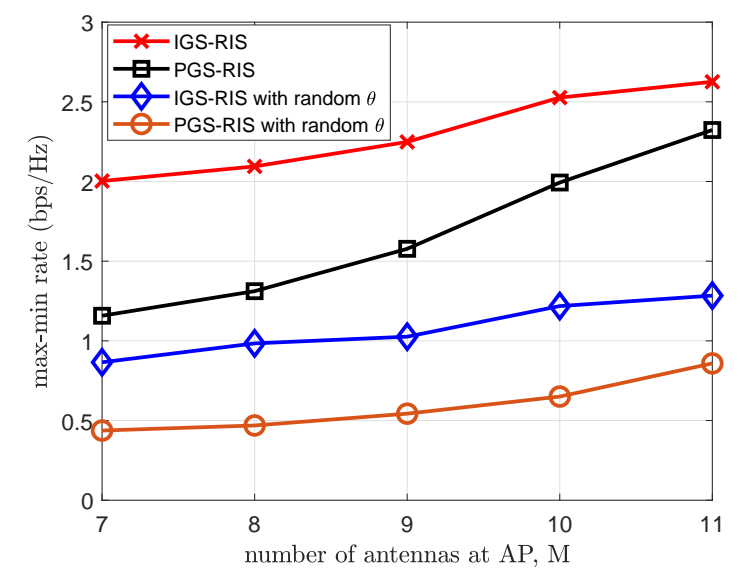

Fig. 8: Assuming blockage of direct path between AP and UEs $h_{a, k} \equiv 0$, achievable max-min rate versus the number of antennas at the AP, $M$, with $P=26 \mathrm{dBm}$ and $N=60$ RIS elements.

Next, we consider another scenario of equally important practice as illustrated by Fig. 7, where there is the blockage of direct signal path between the AP and the multiple UEs, i.e. $h_{a, k} \equiv 0$ in (1) and (5). The path-loss $\beta_{\text {AP-RIS }}$ and $\beta_{\text {RIS-k }}$ are defined by (67a) and (67b). For simulation under this scenario, we can consider slightly smaller distances between AP and the UEs since there is no direct path availability. So under the scenario in Fig. 7, the AP is deployed at $(20,0,25)$, the RIS is deployed at $(0,30,40)$, and $K=10$ UEs are randomly placed in $60 \mathrm{~m} \times 60 \mathrm{~m}$ right-hand-side of the obstacles and RIS.

Fig. 8 plots the achievable max-min rate versus the number of antennas at the AP with $P=26 \mathrm{dBm}$ and $N=60$ RIS elements. Fig. 8 clearly shows that the proposed IGSRIS algorithm outperforms the "IGS-RIS with random $\theta$ " and similarly the proposed PGS-RIS algorithm outperforms the "PGS-RIS with random $\boldsymbol{\theta}$ ". It clearly demonstrates the gain achieved by the proposed algorithms, which consider joint phase optimization with beamforming design over beamforming design alone (random phase selection). Fig. 8 also 
TABLE I: The rounded average number of rounds for implementing Algorithms 1-4 in obtaining Fig. 3 (direct path between the AP and the UEs)

\begin{tabular}{|c|c|c|c|c|c|}
\hline & $M=7$ & $M=8$ & $M=9$ & $M=10$ & $M=11$ \\
\hline IGS-RIS & 36 & 33 & 32 & 34 & 30 \\
\hline IGS-RIS with random $\boldsymbol{\theta}$ & 35 & 31 & 39 & 36 & 33 \\
\hline IGS without RIS & 34 & 33 & 37 & 37 & 37 \\
\hline PGS-RIS & 13 & 14 & 16 & 14 & 14 \\
\hline PGS-RIS with random $\boldsymbol{\theta}$ & 16 & 17 & 24 & 23 & 20 \\
\hline PGS without RIS & 15 & 17 & 24 & 23 & 20 \\
\hline
\end{tabular}

TABLE II: The rounded average number of rounds for implementing Algorithms 1-4 in obtaining Fig.8 (without direct path between the AP and the UEs)

\begin{tabular}{|c|c|c|c|c|c|}
\hline & $M=7$ & $M=8$ & $M=9$ & $M=10$ & $M=11$ \\
\hline IGS-RIS & 53 & 55 & 55 & 56 & 55 \\
\hline PGS-RIS & 41 & 43 & 46 & 49 & 49 \\
\hline IGS-RIS with random $\boldsymbol{\theta}$ & 15 & 15 & 16 & 16 & 16 \\
\hline PGS-RIS with random $\boldsymbol{\theta}$ & 6 & 6 & 6 & 6 & 6 \\
\hline
\end{tabular}

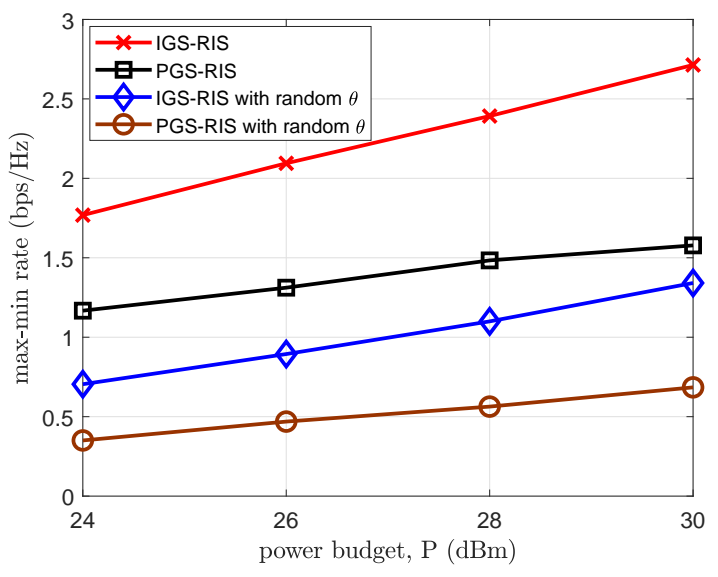

Fig. 9: Assuming blockage of direct path between AP and UEs $h_{a, k} \equiv 0$, achievable max-min rate versus transmit power budget at the AP, $P$, with $M=8$ AP-antennas and $N=60$ RIS elements.

shows the advantage of employing IGS over PGS. Similar trend with superiority of the proposed IGS-RIS algorithm can be observed in Figs. 9 and 10, which plot achievable maxmin rate versus the transmit power budget at the AP and the number of RIS elements, respectively. The above results also show that $\mathbf{w}_{1, k}$ and $\mathbf{w}_{2, k}$ are not one the complex conjugate of the other in IGS-RIS.

Computational experience: To speed up the convergence of Algorithms 2 and 4, at the $\kappa$-th round, define

$$
\mathcal{N}^{(\kappa)} \triangleq\left\{n \in \mathcal{N} \triangleq\{1, \ldots, N\}:\left|\theta_{n}^{(\kappa)}\right|^{2} \geq 1-\epsilon_{\text {tol }}\right\}
$$

for a given tolerance $\epsilon_{t o l}$. Then, replace the trust region constraint (29) in (30), (39), (55), and (65) by the following constraints

$$
\begin{aligned}
& \sum_{n \in \mathcal{N} \backslash \mathcal{N}^{(\kappa)}}\left(2 \Re\left\{\left(\theta_{n}^{(\kappa)}\right)^{*} \theta_{n}\right\}-\left|\theta_{n}^{(\kappa)}\right|^{2}\right)>0, \\
& 2 \Re\left\{\left(\theta_{n}^{(\kappa)}\right)^{*} \theta_{n}\right\}-\left|\theta_{n}^{(\kappa)}\right|^{2} \geq 1-\epsilon_{t o l}, n \in \mathcal{N}^{(\kappa)},
\end{aligned}
$$

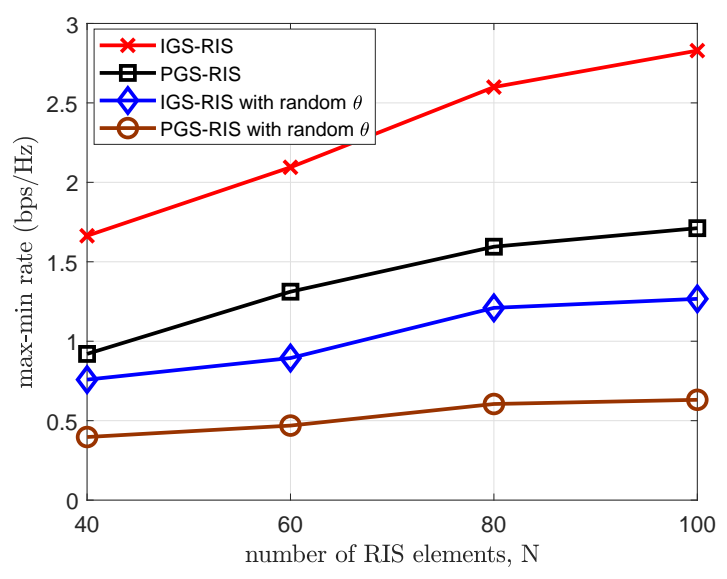

Fig. 10: Assuming blockage of direct path between AP and UEs $h_{a, k} \equiv 0$, achievable max-min rate versus the number of RIS elements, $N$, with $M=8$ AP-antennas and $P=26$ $\mathrm{dBm}$.

to control the convergence of individual $\left|\theta_{n}\right|, n \in \mathcal{N}$ to one.

Table I and Table II provides the rounded average number of rounds in obtaining the numerical results in Fig. 3 (with the direct path between the AP and the UEs) and Fig. 8 (without direct path between the AP and the UEs). In most cases, the second phase of Algorithm 2 and Algorithm 4 takes a couple of iterations to confirm the optimality of the solution found from the first phase. In general, IGS Algorithms 3 and 4 need more rounds than that needed for PGS Algorithms 1 and 2 because optimization of logarithm-determinant functions with IGS is much more computationally challenging than that of logarithmic functions with PGS.

\section{CONCLusions}

The paper has considered a network of an multiple-antenna array access points (AP) serving multiple single-antenna users (UEs) with the assistance of a reconfigured intelligent surface (RIS), under both proper Gaussian signaling (PGS) and 


$$
\begin{aligned}
\left|\mathcal{H}_{k}(\boldsymbol{\theta}) \mathbf{w}_{k}\right|^{2}= & \mathcal{H}_{k}(\boldsymbol{\theta})\left[\mathbf{w}_{k}\right]^{2} \mathcal{H}_{k}^{H}(\boldsymbol{\theta}) \\
= & \mathcal{H}_{k}(\boldsymbol{\theta})\left(\left[\mathbf{w}_{k}\right]^{2}+\eta I_{M}\right) \mathcal{H}_{k}^{H}(\boldsymbol{\theta})-\eta\left[\mathcal{H}_{k}(\boldsymbol{\theta})\right]^{2} \\
\geq & \mathcal{H}_{k}\left(\theta^{(\kappa)}\right) Y_{k}^{(\kappa)}(\eta) \mathcal{H}_{k}^{H}(\boldsymbol{\theta})+\mathcal{H}_{k}(\boldsymbol{\theta}) Y_{k}^{(\kappa)}(\eta)\left(\mathcal{H}_{k}^{H}\left(\theta^{(\kappa)}\right)\right. \\
& -\mathcal{H}_{k}\left(\theta^{(\kappa)}\right) Y_{k}^{(\kappa)}(\eta)\left(\left[\mathbf{w}_{k}\right]^{2}+\eta I_{M}\right)^{-1} Y_{k}^{(\kappa)}(\eta) \mathcal{H}_{k}^{H}\left(\theta^{(\kappa)}\right)-\eta\left[\mathcal{H}_{k}(\boldsymbol{\theta})\right]^{2} \\
\geq & \mathcal{H}_{k}\left(\theta^{(\kappa)}\right) Y_{k}^{(\kappa)}(\eta) \mathcal{H}_{k}^{H}(\boldsymbol{\theta})+\mathcal{H}_{k}(\boldsymbol{\theta}) Y_{k}^{(\kappa)}(\eta) \mathcal{H}_{k}^{H}\left(\theta^{(\kappa)}\right) \\
& -\mathcal{H}_{k}\left(\theta^{(\kappa)}\right) Y_{k}^{(\kappa)}(\eta)\left(w_{k}^{(\kappa)} \mathbf{w}_{k}^{H}+\mathbf{w}_{k}\left(w_{k}^{(\kappa)}\right)^{H}-\left[w_{k}^{(\kappa)}\right]^{2}+\eta I_{M}\right)^{-1} \\
& \times Y_{k}^{(\kappa)}(\eta) \mathcal{H}_{k}^{H}\left(\theta^{(\kappa)}\right)-\eta\left\langle\boldsymbol{X}_{k}\right\rangle .
\end{aligned}
$$

improper Gaussian signaling (IGS) with and without direct channels from the AP to UEs. The problem of jointly designing the RIS's reflecting coefficients and transmit beamformers to maximize the users' worst rate subject to the transmit power constraint has been addressed. Namely, the paper has developed algorithms of low computational complexity, which converge at least to a locally optimal solution. The provided simulations have shown the clear advantage of IGS over PGS, and of RIS-aided links over RIS-less links. Their extensions to similar problems for multiple-antenna users are under current study. Another future research direction is to consider channel estimation and solve the joint design of RIS's reflecting coefficients and transmit beamformers in the presence of channel estimation errors.

\section{APPENDIX A: FUNDAMENTAL INEQUALITIES}

The following results of [46] are used

$$
\frac{\mathbf{x}}{\mathbf{y}} \geq \frac{3 \bar{x}}{\bar{y}}-\frac{\bar{x}^{2}}{\bar{y}} \frac{1}{\mathbf{x}}-\frac{\bar{x}}{\bar{y}^{2}} \mathbf{y} \quad \forall(\mathbf{x}, \mathbf{y}) \in \mathbb{R}_{+}^{2} \&(\bar{x}, \bar{y}) \in \mathbb{R}_{+}^{2},
$$

and

$$
\begin{aligned}
\frac{|\boldsymbol{x}|^{2}}{\mathbf{y}} & \geq \frac{2 \Re\left\{\bar{x}^{*} \boldsymbol{x}\right\}}{\bar{y}}-\frac{|\bar{x}|^{2}}{\bar{y}^{2}} \mathbf{y} \\
& \forall(\boldsymbol{x}, \mathbf{y}) \in \mathbb{C} \times \mathbb{R}_{+} \&(\bar{x}, \bar{y}) \in \mathbb{C} \times \mathbb{R}_{+},
\end{aligned}
$$

where $\mathbb{R}_{+}^{m} \triangleq\left\{\left(x_{1}, \ldots, x_{m}\right): x_{i}>0, i=1, \ldots, m\right\}$.

The following inequalities for matrices of dimension $2 \times 2$ hold true [47], [48]:

$$
\begin{array}{r}
\ln \left|I_{2}+[\mathbf{V}]^{2}(\mathbf{Y})^{-1}\right| \geq \ln \left|I_{2}+[\bar{V}]^{2}(\bar{Y})^{-1}\right| \\
-\left\langle[\bar{V}]^{2}(\bar{Y})^{-1}\right\rangle+2 \Re\left\{\left\langle\bar{V}^{H}(\bar{Y})^{-1} \mathbf{V}\right\rangle\right\} \\
-\left\langle(\bar{Y})^{-1}-\left(\bar{Y}+[\bar{V}]^{2}\right)^{-1},[\mathbf{V}]^{2}+\mathbf{Y}\right\rangle, \\
\forall \mathbf{V}, \mathbf{Y} \succ 0 \& \bar{V}, \bar{Y} \succ 0,
\end{array}
$$

and

$$
\begin{aligned}
\ln \mid \boldsymbol{X}^{-1}+ & \mathbf{Y}^{-1}|\geq \ln | \bar{X}^{-1}+\bar{Y}^{-1} \mid+2 \\
& -\left\langle(\bar{X})^{-1}-(\bar{X}+\bar{Y})^{-1}, \boldsymbol{X}\right\rangle \\
& -\left\langle(\bar{Y})^{-1}-(\bar{X}+\bar{Y})^{-1}, \mathbf{Y}\right\rangle \\
\forall \boldsymbol{X} \succ & \succ, \mathbf{Y} \succ 0 \& \bar{X} \succ 0, \bar{Y} \succ 0,
\end{aligned}
$$

and

$\ln |\boldsymbol{X}| \geq \ln |\bar{X}|+2-\left\langle\bar{X},(\boldsymbol{X})^{-1}\right\rangle \forall \boldsymbol{X} \succ 0 \& \bar{X} \succ 0$.
The following matrix inequality holds true for all matrices $\mathbf{Y} \succ 0, \bar{Y} \succ 0$ and $\boldsymbol{X}$ and $\bar{X}$ of appropriate dimension [49, Appendix C]

$$
\boldsymbol{X} \mathbf{Y} \boldsymbol{X}^{H} \succeq \bar{X} \bar{Y} \boldsymbol{X}^{H}+\boldsymbol{X} \bar{Y} \bar{X}^{H}-\bar{X} \bar{Y} \mathbf{Y}^{-1} \bar{Y} \bar{X}^{H} .
$$

Theorem 1: The following inequality holds true for all $\boldsymbol{X} \succ$ $0, \mathbf{Y} \succ 0$ and $\bar{X} \succ 0, \bar{Y} \succ 0$ :

$$
\langle\boldsymbol{X}, \mathbf{Y}\rangle \leq \frac{1}{2}\left(\left\|\bar{X}^{-1 / 2} \boldsymbol{X} \bar{Y}^{1 / 2}\right\|^{2}+\left\|\bar{X}^{1 / 2} \mathbf{Y} \bar{Y}^{-1 / 2}\right\|^{2}\right)
$$

Proof: From the inequality

$$
\begin{aligned}
\left(\bar{X}^{-1 / 2} \boldsymbol{X} \bar{Y}^{1 / 2}-\bar{X}^{1 / 2} \mathbf{Y} \bar{Y}^{-1 / 2}\right) & \left(\bar{X}^{-1 / 2} \boldsymbol{X} \bar{Y}^{1 / 2}-\bar{X}^{1 / 2} \mathbf{Y} \bar{Y}^{-1 / 2}\right)^{H} \succeq 0
\end{aligned}
$$

one has

$$
\begin{gathered}
\bar{X}^{-1 / 2} \boldsymbol{X} \bar{Y}^{1 / 2} \bar{Y}^{-1 / 2} \mathbf{Y} \bar{X}^{1 / 2} \\
+\bar{X}^{1 / 2} \mathbf{Y} \bar{Y}^{-1 / 2} \bar{Y}^{1 / 2} \boldsymbol{X} \bar{X}^{-1 / 2} \\
\preceq \bar{X}^{-1 / 2} \boldsymbol{X} \bar{Y}^{1 / 2}\left(\bar{X}^{-1 / 2} \boldsymbol{X} \bar{Y}^{1 / 2}\right)^{H} \\
+\bar{X}^{1 / 2} \mathbf{Y} \bar{Y}^{-1 / 2}\left(\bar{X}^{1 / 2} \mathbf{Y} \bar{Y}^{-1 / 2}\right)^{H} .
\end{gathered}
$$

Therefore,

$$
\begin{aligned}
2\langle\boldsymbol{X Y}\rangle & =\left\langle\bar{X}^{-1 / 2} \boldsymbol{X} \bar{Y}^{1 / 2} \bar{Y}^{-1 / 2} \mathbf{Y} \bar{X}^{1 / 2}\right\rangle \\
& +\left\langle\bar{X}^{1 / 2} \mathbf{Y} \bar{Y}^{-1 / 2} \bar{Y}^{1 / 2} \boldsymbol{X} \bar{X}^{-1 / 2}\right\rangle \\
\leq & \left\|\bar{X}^{-1 / 2} \boldsymbol{X} \bar{Y}^{1 / 2}\right\|^{2}+\left\|\bar{X}^{1 / 2} \mathbf{Y} \bar{Y}^{-1 / 2}\right\|^{2}
\end{aligned}
$$

which is (78).

\section{APPENDIX B: PROOF FOR (27)}

Using the inequality (77) in the appendix A yields (71) on the top of this page. Therefore, the nonconvex constraint (36) is innerly approximated by the constraint

$$
\text { RHS of (71) } \geq \mathbf{z}_{k},
$$

which is

$$
\begin{aligned}
\left(\mathcal{H}_{k}\left(\theta^{(\kappa)}\right)\right. & \left.Y_{k}^{(\kappa)}(\eta) \mathcal{H}_{k}^{H}(\boldsymbol{\theta})+(*)^{H}\right)-\mathbf{z}_{k}-\eta\left\langle\boldsymbol{X}_{k}\right\rangle \\
& -\mathcal{H}_{k}\left(\theta^{(\kappa)}\right) Y_{k}^{(\kappa)}(\eta)\left[\left(w_{k}^{(\kappa)} \mathbf{w}_{k}^{H}+(*)^{H}\right)\right. \\
& \left.-\left[w_{k}^{(\kappa)}\right]^{2}+\eta I_{M}\right]^{-1} Y_{k}^{(\kappa)}(\eta) \mathcal{H}_{k}^{H}\left(\theta^{(\kappa)}\right) \geq 0 .
\end{aligned}
$$

The latest inequality is equivalent to (27) by the Shur's complement. 


\section{REFERENCES}

[1] J. G. Andrews, S. Buzzi, W. Choi, S. V. Hanly, A. Lozano, A. C. K. Soong, and J. C. Zhang, "What will 5G be?", IEEE J. Sel. Areas Commun., vol. 32, pp. 1065-1082, Jun. 2014.

[2] F. Boccardi, R. W. Heath, A. Lozano, T. L. Marzetta, and P. Popovski, "Five disruptive technology directions for 5G," IEEE Commun. Mag., vol. 52, pp. 74-80, Feb. 2014.

[3] S. Zhang, Q. Wu, S. Xu, and G. Y. Li, "Fundamental green tradeoffs: Progresses, challenges, and impacts on 5G networks," IEEE Commun. Surveys Tuts., vol. 19, pp. 33-56, Firstquarter 2017.

[4] Q. Wu, G. Y. Li, W. Chen, D. W. K. Ng, and R. Schober, "An overview of sustainable green 5G networks," IEEE Wirel. Commun., vol. 24, pp. 72 80, Aug. 2017.

[5] S. Buzzi, C.-L. I, T. E. Klein, H. V. Poor, C. Yang, and A. Zappone, "A survey of energy-efficient techniques for $5 \mathrm{G}$ networks and challenges ahead," IEEE J. Select. Areas Commun., vol. 34, pp. 697-709, Apr. 2016.

[6] C. Huang, A. Zappone, G. C. Alexandropoulos, M. Debbah, and C. Yuen, "Reconfigurable intelligent surfaces for energy efficiency in wireless communication," IEEE Trans. Wirel. Commun., vol. 18 , pp. 4157-4170, Aug. 2019.

[7] Q. Wu and R. Zhang, "Towards smart and reconfigurable environment Intelligent reflecting surface aided wireless network," IEEE Commun. Mag., vol. 58, pp. 106-112, Jan. 2020.

[8] E. Basar, M. Di Renzo, J. De Rosny, M. Debbah, M.-S. Alouini, and R. Zhang, "Wireless communications through reconfigurable intelligent surfaces," IEEE Access, vol. 7, pp. 116753-116773, Sept. 2019

[9] S. Tretyakov, V. Asadchy, and A. Diaz-Rubio, "Chapter 6: Metasurfaces for general control of reflection and transmission," in World Scientific Handbook of Metamaterials and Plasmonics, pp. 249-293, 2017.

[10] F. Liu et al., "Intelligent metasurfaces with continuously tunable local surface impedance for multiple reconfigurable functions," Phys. Rev. Appl., vol. 11, no. 4, p. 10, 2019.

[11] Z. Li, Y. Xie, L. Shangguan, R. I. Zelaya, J. Gummeson, W. Hu, and $\mathrm{K}$. Jamieson, "Towards programming the radio environment with large arrays of inexpensive antennas," in Proc. 16th USENIX Symp. Netw. Syst. Design Implement. (NSDI 19), pp. 285-300, Feb. 2019.

[12] M. Di Renzo, M. Debbah, D.-T. Phan-Huy, A. Zappone, M.-S. Alouini, C. Yuen, V. Sciancalepore, G. C. Alexandropoulos, J. Hoydis, H. Gacanin, J. de Rosny, A. Bounceu, G. Lerosey, and M. Fink, "Smart radio environments empowered by AI reconfigurable meta-surfaces: An idea whose time has come," EURASIP J. Wirel. Commun. Network. pp. 1-20, May. 2019.

[13] S. Gong, J. Xu, D. Niyato, X. Huang, and Z. Han, "Backscatter-aided cooperative relay communications in wireless-powered hybrid radio networks," IEEE Network, vol. 33, pp. 234-241, Sept. 2019.

[14] D. Ciuonzo, G. Gelli, A. Pescapé, and F. Verde, "Decision fusion rules in ambient backscatter wireless sensor networks," in Proc. 2019 IEEE 30th Ann. Inter. Symp. Pers. Ind. Mob. Radio Commun. (PIMRC), pp. 1-6, IEEE, 2019.

[15] S. Hu, F. Rusek, and O. Edfors, "Beyond massive MIMO: The potential of data transmission with large intelligent surfaces," IEEE Trans. Signal Process., vol. 66, pp. 2746-2758, May. 2018.

[16] K. Ntontin, M. Di Renzo, J. Song, F. Lazarakis, J. de Rosny, D.-T Phan-Huy, O. Simeone, R. Zhang, M. Debbah, G. Lerosey, M. Fink, S. Tretyakov, and S. Shama, "Reconfigurable intelligent surfaces vs. relaying: Differences, similarities, and performance comparison," https://arxiv.org/abs/1908.08747.

[17] Q. Wu and R. Zhang, "Intelligent reflecting surface enhanced wireless network via joint active and passive beamforming," IEEE Trans. Wirel. Commun., vol. 18, pp. 5394-5409, Nov. 2019.

[18] Q.-U.-A. Nadeem, A. Kammoun, A. Chaaban, M. Debbah, and M.-S. Alouini, "Asymptotic max-min SINR analysis of reconfigurable intelligent surface assisted MISO systems," https://arxiv.org/pdf/1903.08127.pdf.

[19] A. H. Phan, H. D. Tuan, H. H. Kha, and D. T. Ngo, "Nonsmooth optimization for efficient beamforming in cognitive radio multicast transmission," IEEE Trans. Signal Process., vol. 60, pp. 2941-2951, Jun. 2012.

[20] G. Zhou, C. Pan, H. Ren, K. Wang, W. Xu, and A. Nallanathan, "Intelligent reflecting surface aided multigroup multicast MISO communication systems," https://arxiv.org/abs/1909.04606.

[21] C. Pan, H. Ren, K. Wang, W. Xu, M. Elkashlan, A. Nallanathan, and L. Hanzo, "Multicell MIMO communications relying on intelligent reflecting surface," https://arxiv.org/abs/1907.10864.
[22] C. Pan, H. Ren, K. Wang, M. Elkashlan, A. Nallanathan, J. Wang, and L. Hanzo, "Intelligent reflecting surface aided MIMO broadcasting for simultaneous wireless information and power transfer," IEEE J. Select. Areas Commun. (Accepted).

[23] H. Shin, S. Park, H. Park, and I. Lee, "A new approach of interference alignment through asymmetric complex signaling and multiuser diversity," IEEE Trans. Wirel. Commun., vol. 11, pp. 880-884, Mar. 2012.

[24] C. Hellings, M. Joham, and W. Utschick, "QoS feasibility in MIMO broadcast channels with widely linear transceivers," IEEE Signal Process. Letts., vol. 20, pp. 1134-1137, Nov. 2013.

[25] S. Lagen, A. Agustin, and J. Vidal, "Coexisting linear and widely linear transceivers in the MIMO interference channel," IEEE Trans. Signal Process., vol. 64, pp. 652-664, Feb. 2016.

[26] A. A. Nasir, H. D. Tuan, T. Q. Duong, and H. V. Poor, "Improper Gaussian signaling for broadcast interference networks," IEEE Signal Process. Lett., vol. 26, pp. 808-812, Jun. 2019.

[27] H. D. Tuan, A. A. Nasir, H. H. Nguyen, T. Q. Duong, and H. V. Poor, "Non-orthogonal multiple access with improper Gaussian signaling," IEEE J. Selec. Topics Signal Process., vol. 13, pp. 496-507, Mar. 2019.

[28] H. Yu, H. D. Tuan, T. Q. Duong, Y. Fang, and L. Hanzo, "Improper Gaussian signaling for integrated data and energy networking," IEEE Trans. Commun. (Early Access), 2020

[29] G. Gelli, L. Paura, and A. R. Ragozini, "Blind widely linear multiuser detection," IEEE Commun. Lett., vol. 4, pp. 187-189, Jun. 2000.

[30] S. Buzzi, M. Lops, and S. Sardellitti, "Widely linear reception strategies for layered space-time wireless communications," IEEE Trans. Signal Process., vol. 54, pp. 2252-2262, Jun. 2006.

[31] P. J. Schrerier and L. L. Scharf, Statistical Signal Processing of ComplexValued Data: The Theory of Improper and Noncircular Signals. Cambridge University Press, 2010.

[32] O. Ozdogan, E. Bjornson, and E. G. Larsson, "Intelligent reflecting surfaces: Physics, propagation, and pathloss modeling," https://arxiv.org/abs/1911.03359.

[33] Q.-U.-A. Nadeem, A. Kammoun, M. Debbah, and M.-S. Alouini, "A generalized spatial correlation model for 3D MIMO channels based on the Fourier coefficients of power spectrums," IEEE Trans. Signal Process., vol. 63, pp. 3671-3686, Jul. 2015.

[34] E. Bjornson, O. Ozdogan, and E. G. Larsson, "Intelligent reflecting surface vs. decode-and-forward: How large surfaces are needed to beat relaying?," IEEE Wirel. Commun. Lett., vol. 9, pp. 244-248, Feb. 2020.

[35] A. A. Nasir, H. D. Tuan, D. T. Ngo, T. Q. Duong, and H. V. Poor, "Beamforming design for wireless information and power transfer systems: Receive power-splitting versus transmit time-switching," IEEE Trans. Commun., vol. 65, pp. 876-889, Feb. 2017.

[36] A. A. Nasir, H. D. Tuan, T. Q. Duong, and H. V. Poor, "Secrecy rate beamforming for multicell networks with information and energy harvesting," IEEE Trans, Signal Process., vol. 65, pp. 677-689, Feb. 2017.

[37] A. Wiesel, Y. C. Eldar, and S. Shamai, "Linear precoding via conic optimization for fixed MIMO receivers," IEEE Trans. Signal Process. vol. 54, pp. 161-176, Jan. 2006.

[38] H. Tuy, Convex Analysis and Global Optimization (second edition). Springer International, 2017.

[39] E. Che, H. D. Tuan, and H. H. Nguyen, "Joint optimization of cooperative beamforming and relay assignment in multi-user wireless relay networks," IEEE Trans. Wirel. Commun., vol. 13, pp. 5481-5495, Oct. 2014.

[40] H. H. M. Tam, H. D. Tuan, D. T. Ngo, T. Q. Duong, and H. V. Poor, "Joint load balancing and interference management for small-cell heterogeneous networks with limited backhaul capacity," IEEE Trans. Wirel. Commun., vol. 16, pp. 872-884, Feb. 2017.

[41] Y. Shi, H. D. Tuan, T. Q. Duong, H. V. Poor, and A. V. Savkin, "PMU placement optimization for efficient state estimation in smart grid," IEEE J. Select. Areas Commun., vol. 38, pp. 71-83, Jan. 2019.

[42] J. F. Bonnans, J. C. Gilbert, C. Lemarechal, and C. Sagastizabal, Numerical Optimization: Theoretical and Practical Aspects (second edition). Springer, 2006.

[43] D. Peaucelle, D. Henrion, and Y. Labit, "Users guide for SeDuMi interface 1.03," 2002.

[44] Y. Shi, H. D. Tuan, S. Su, and H. Tuy, "Global optimization for optimal power flow over transmission networks," J. Global Optimiz., vol. 69 , pp. 745-760, Nov. 2017.

[45] I. E. Telatar, "Capacity of multi-antenna Gaussian channels," Eur. Trans. Telecommun., vol. 10, pp. 585-595, Nov./Dec. 1999.

[46] Z. Sheng, H. D. Tuan, T. Q. Duong, H. V. Poor, and Y. Fang, "Lowlatency multiuser two-way wireless relaying for spectral and energy 
efficiencies," IEEE Trans. Signal Process., vol. 66, pp. 4362-4376, Aug. 2018.

[47] H. H. M. Tam, H. D. Tuan, and D. T. Ngo, "Successive convex quadratic programming for quality-of-service management in full-duplex MUMIMO multicell networks," IEEE Trans. Commun., vol. 64, pp. 23402353, Jun. 2016.

[48] A. A. Nasir, H. D. Tuan, T. Q. Duong, and M. Debbah, "NOMA throughput and energy efficiency in energy harvesting enabled networks," IEEE Trans. Commun., vol. 67, pp. 6499-6511, Sept. 2019.

[49] U. Rashid, H. D. Tuan, H. H. Kha, and H. H. Nguyen, "Joint optimization of source precoding and relay beamforming in wireless MIMO relay networks," IEEE Trans. Commun., vol. 62, pp. 488-499, Feb. 2014.

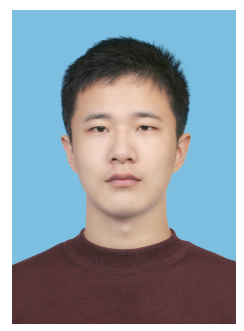

Hongwen Yu received the B.S. degree in Communication and Information Engineering from the Shanghai University, Shanghai, China, in 2011, and the M.S. degree in Communication and Information Engineering from the Shanghai University, Shanghai, China, in 2014. He is currently pursuing the $\mathrm{Ph} . \mathrm{D}$. degree with the School of Electrical and Data Engineering, University of Technology Sydney, Ultimo, NSW, Australia, and with Shanghai University, Shanghai, China. His current research interests include optimization methods for wireless communication and signal processing.

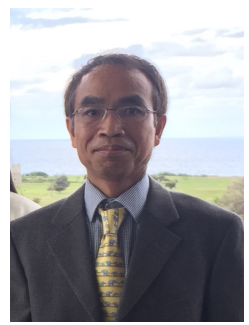

Hoang Duong Tuan received the Diploma (Hons.) and Ph.D. degrees in applied mathematics from Odessa State University, Ukraine, in 1987 and 1991, respectively. He spent nine academic years in Japan as an Assistant Professor in the Department of Electronic-Mechanical Engineering, Nagoya University, from 1994 to 1999, and then as an Associate Professor in the Department of Electrical and Computer Engineering, Toyota Technological Institute, Nagoya, from 1999 to 2003. He was a Professor with the School of Electrical Engineering and Telecommunications, University of New South Wales, from 2003 to 2011. He is currently a Professor with the School of Electrical and Data Engineering, University of Technology Sydney. He has been involved in research with the areas of optimization, control, signal processing, wireless communication, and biomedical engineering for more than 20 years.

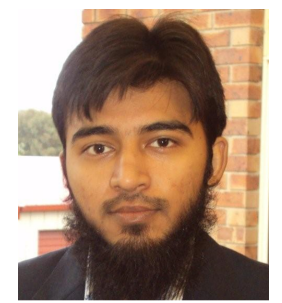

Ali Arshad Nasir received his Ph.D. in telecommunications engineering from the Australian National University (ANU), Australia in 2013 and worked there as a Research Fellow from until 2015. From 2015 to 2016, he was an Assistant Professor in the School of Electrical Engineering and Computer Science (SEECS) at National University of Sciences \& Technology (NUST), Pakistan. Currently, he is an Assistant Professor in the Department of Electrical Engineering, King Fahd University of Petroleum and Minerals (KFUPM), Dhahran, KSA. His research interests are in the area of signal processing in wireless communication systems. He is an Associate Editor for IEEE Canadian Journal of Electrical and Computer Engineering.

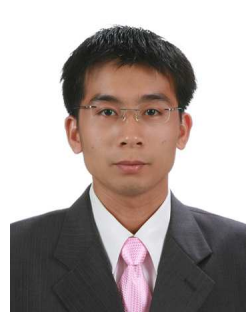

Trung Q. Duong (S'05, M'12, SM'13) received his $\mathrm{Ph} . \mathrm{D}$. degree in Telecommunications Systems from Blekinge Institute of Technology (BTH), Sweden in 2012. Currently, he is with Queen's University Belfast (UK), where he was a Lecturer (Assistant Professor) from 2013 to 2017 and a Reader (Associate Professor) from 2018. His current research interests include wireless communications, machine learning, realtime optimisation, big data, and IoT applications to disaster management, airquality monitoring, flood monitoring, smart agriculture, healthcare and smart cities. He is the author or co-author of over 350+ technical papers published in scientific journals $(220+$ articles $)$ and presented at international conferences (140+ papers).

Dr. Duong currently serves as an Editor for the IEEE TRANSACTIONS ON WiRELESS COMMUNiCATIONS, IEEE TRANSACTIONS ON COMMUNICATIONS, and an Executive Editor for IEEE COMMUNiCATIONS LETTERS. He was awarded the Best Paper Award at the IEEE Vehicular Technology Conference (VTC-Spring) in 2013, IEEE International Conference on Communications (ICC) 2014, IEEE Global Communications Conference (GLOBECOM) 2016 and 2019, IEEE Digital Signal Processing Conference (DSP) 2017, and International Wireless Communications \& Mobile Computing Conference (IWCMC) 2019. He is the recipient of prestigious Royal Academy of Engineering Research Fellowship (2016-2021) and has won a prestigious Newton Prize 2017.

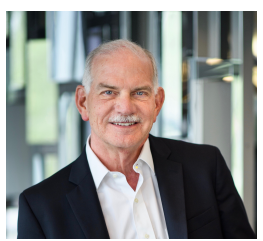

H. Vincent Poor (S72, M77, SM82, F87) received the Ph.D. degree in EECS from Princeton University in 1977. From 1977 until 1990, he was on the faculty of the University of Illinois at Urbana-Champaign. Since 1990 he has been on the faculty at Princeton, where he is currently the Michael Henry Strater University Professor of Electrical Engineering. During 2006 to 2016, he served as Dean of Princeton's School of Engineering and Applied Science. He has also held visiting appointments at several other universities, including most recently at Berkeley and Cambridge. His research interests are in the areas of information theory, signal processing and machine learning, and their applications in wireless networks, energy systems and related fields. Among his publications in these areas is the recent book Multiple Access Techniques for $5 G$ Wireless Networks and Beyond (Springer, 2019).

Dr. Poor is a member of the National Academy of Engineering and the National Academy of Sciences, and is a foreign member of the Chinese Academy of Sciences, the Royal Society, and other national and international academies. Recent recognition of his work includes the 2017 IEEE Alexander Graham Bell Medal and a D.Eng. honoris causa from the University of Waterloo awarded in 2019. 